\title{
Cognitive Choice Processes and the Attitude-Behavior Relation
}

\author{
Dietrich Albert, K. Michael Aschenbrenner, and \\ Franz Schmalhofer
}

Fishbein and Ajzen's (1975) theory of reasoned action is currently the most successful and much discussed theory in attitude-behavior research. Although the theory is well established for predicting behavior in various contexts, its central assumption of a static attitude-behavior intention relation appears questionable when recent results of cognitively oriented decision research are considered. This assumption is dynamically reformulated in order to model the cognitive processes in binary choice. The resulting criterion-dependent choice models assume a sequential evaluative processing of the alternatives' attributes until enough evidence in favor of one alternative is accumulated. Within this general framework, specific models distinguish between internally (memory) and externally (display) available information about the alternatives. The models and a series of experimental studies, which were specifically designed to investigate various aspects of the models, are comprehensively presented. Relations to other theories and consequences for attitude-behavior research are discussed.

Fishbein and Ajzen's Contribution to the Attitude-Behavior Problem

According to Stroebe (1980, p. 138), at the start of the 1970s social psychology went through a difficult period because of the attitude-behavior inconsistencies. The resolution of this crisis is credited in particular to the efforts of Fishbein and Ajzen. By means of their principle of correspondence (Ajzen \& Fishbein, 1977; Fishbein, 1973; Fishbein \& Ajzen, 1974), they ordered the heterogeneous findings (e.g., Wicker, 1969) on the attitude-behavior relation which caused this crisis.

Further, they made a successful proposal on how behavior depends on attitudes. This was done in their theory of reasoned action (Ajzen \& Fishbein, 
1980; Fishbein \& Ajzen, 1975), which was recently extended into the theory of planned action (Ajzen, 1985).

Their theory's central assumptions regarding the effects of attitudes are embodied in two equations (see equations (1) and (2); Fishbein \& Ajzen, 1975, p. $301)$.

$$
B \sim I=\left(A_{B}\right) w+\text { other variable(s), }
$$

where $B=$ the behavior in question,

$I=$ the person's intention to perform behavior $B$,

$A_{B}=$ the attitude (evaluation) toward performing behavior $B$,

$w=$ a regression weight.

According to Fishbein $(1963,1967)$, the attitude formation is conceptualized as an expectancy times value model or-more generally-as a bilinear model.

$$
A_{B}=\sum_{i=1}^{n} b_{i} e_{i}
$$

where $b_{i}=$ the strength of the salient belief (subjective probability) held by a person that performing behavior $B$ leads to outcome $i$ ("outcome" includes consequences, effort, costs, characteristics and other attributes);

$e_{i}=$ the evaluation (attitude) of outcome $i$;

$n=$ the number of salient beliefs the person actually holds regarding performing behavior $B$.

Ajzen and Fishbein (1980) present their model as a process model in the sense of a causal chain, in which the "salient beliefs are the immediate determinants of the person's attitude" (p. 63). This attitude is one of the antecedents of the intention that is the immediate determinant of the behavioral act. However, their theory has only been tested by regression methods and correlational statistics. According to the principle of correspondence the values $I, A_{b}, b$, and $e$ are elicited by individual-rating procedures. However, the beliefs used are those salient in a given population (the so-called modal salient beliefs) or the first few beliefs (five to nine) of an individual, which in both cases are elicited by a free response task (continuous association). Thus Ajzen and Fishbein did not solve the problem of determining the salient beliefs a person actually holds. One possibility is to ask a person for his beliefs after the formation of his intention, as by Westhoff (Chapter 8, this volume). Other possibilities for solving the problem, by specific methods of observation or by the application of process models, are presented in the two sections after the next one. 


\section{The Theory of Reasoned Action, A Process Model?}

Discussing the theory of reasoned action (and its derivatives not mentioned here) is difficult because of Fishbein and Ajzen's intention-behavior gap. They intend to consider salient beliefs ("in order to predict a person's attitude, it is not sufficient to know what information he has been given; rather it is necessary to assess the beliefs he actually holds, i.e. his salient beliefs." Fishbein \& Ajzen, 1975, p. 233; "in order to understand why a person holds a certain attitude toward an object it is necessary to assess his salient beliefs about that object." Ajzen \& Fishbein, 1980, p. 63), but in actuality they do not: "It appears impossible to obtain a precise measure of the beliefs that determine an individual's attitude since the number of salient beliefs may vary from person to person" (Fishbein \& Ajzen, 1975, p. 219). They intend to test a process model of the attitude-behavior relation ("we are again advocating an approach which explicates the processes intervening between stimulus and response variables," p. 334), but they investigate a regression model (Ajzen \& Fishbein, 1980, pp. 98-100). The question of which part of their theory was to be discussed had to be decided. We decided to discuss that part of the theory which applied to data, because Ajzen and Fishbein (1980) refer to this part when they conclude that "some people may arrive at their decisions in different ways. The accumulating evidence suggests, however, that the theory is useful for most individuals and with respect to most social behaviors" (p. 245).

Their theory is an excellent contribution to the prediction of behavior. However, we disagree with its use "as a description of human information processing" (p. 245) for the following reasons:

1. The predictability of a more or less specific behavior-intention judgment from attitudinal and subjective-norm judgments by means of a linear model (equation 1) does not prove that behavioral attitudes control behavioral intentions (cf. Birnbaum, 1973; Dawes, 1979; Dawes \& Corrigan, 1974; Einhorn, Kleinmuntz, \& Kleinmuntz, 1979).

2. The predictability of a behavior-attitude judgment from evaluative and belief-strength judgments by a bilinear model (equation 2 ) does not prove that these variables control attitudinal judgments in that way (Busemeyer \& Jones, 1983; Thorngate, 1980).

3. It appears doubtful that the variable "belief strength" played any role at all in the behavioral intentions or attitude formations that were investigated by Fishbein and Ajzen. The variance-explaining effect of this variable may have been an artifact of the methods used (cf. Cronen \& Conville, 1975; Delia, Crockett, Press, \& O’Keefe, 1975).

Of course, arguments 1 through 3 do not exclude the possibility that some behavioral intentions are determined by a holistic attitude (equation 1), and that some holistic attitudes evolve according to the bilinear model (equation 
2). However, it is doubtful that this is very often the case for the following reason, which is our main argument:

4. Detailed analyses of decision and choice behavior have shown that (a) behavior is often determined by heuristic processing of individual attributes of objects that does not result in holistic evaluations of these objects (e.g., Russo \& Dosher, 1983), (b) holistic judgments are often determined by heuristics that are incompatible with a bilinear model (e.g., Schoemaker, 1979), (c) judgmental and choice processes often differ (Lichtenstein \& Slovic, 1971) in such ways that the first cannot be part of the second, and (d) especially expectancy times value models cannot explain choice behavior (Aschenbrenner, 1984, 1985).

The results of cognitive decision research suggest a modification and an extension of the processing part of Fishbein and Ajzen's theory. The methods that were developed and used in cognitive decision research (cf. Aschenbrenner, 1979) allow additional insights into the processes that precede behavioral choice. Analyzing these processes is exactly what Fishbein and Ajzen originally intended to do.

\section{Principles for Analyzing Cognitive Choice Processes}

On a global level of theorizing, agreement can be made with Ajzen and Fishbein $(1980$, p. 41$)$ "that all behavior involves a choice, be it a choice between performing or not performing a given action or a choice among several qualitatively or quantitatively different action alternatives." Thus it appears natural that the process components that were postulated by Fishbein and Ajzen are easily assigned to the states of a choice process.

On the basis of cognitive choice research the components of a choice process may be summarized: (1) becoming aware of a decision problem and/or realizing an (unsatisfied) behavioral objective; (2) selecting information about features of potential behavioral alternatives with respect to situation and objective; (3) evaluating the selected information with respect to situation and/or objective and aggregating these evaluations; (4) termination of information selection, evaluation, aggregation, and finally choice of behavior.

Within this framework, predicting and describing individual choice may fail for the following reasons (most of the following principles are also in accordance with the theory of reasoned action, thus demonstrating that a convergence between attitude and choice research has also been reached on an empirical level):

1. A person may perceive the situation differently or may pursue different objectives than those assumed by the experimenter. In our experiments situations and objectives will be prescribed by instruction.

2. A person may consider other behavioral alternatives than those assumed 
by the experimenter. Hence we will inquire about potential behavioral alternatives or define the set of alternatives.

3. A person may select other information about the alternatives than the experimenter assumes. Consequently, we will inquire about the alternatives' features, define the features, or make the selection process observable.

4. A person may evaluate the alternatives' features differently than the experimenter. Therefore, we will inquire about the evaluations or prescribe them.

5. A person may aggregate the component judgments differently than the experimenter assumes. Hence we will test specific predictions of our favorite aggregation rule and consider other rules additionally.

6. Before terminating the choice process a person may process a different amount of information than the experimenter assumes. Therefore, we will observe the latency of decision as well as the processed informationwhenever possible.

We tried not only to consider these principles as guidelines for our empirical proceeding; but also attempted to capture their aspects of selectivity, flexibility, and adaptivity in the development of our theoretical framework; the so-called criterion-dependent choice (CDC) models.

\section{General Method of Empirical Investigation}

Typically our studies consisted of two kinds of tasks: choice tasks and evaluation tasks. The order of these sections was varied. No systematic effect of this variation was observed.

\section{The Choice Tasks}

So far, our research considered choices between two alternatives at a time, that is, binary choices. Subjects were instructed to imagine a choice situation (according to principle 1). For example, choosing journal subscriptions which are offered as a birthday present. A choice pair was then presented and the subjects had to decide which of the two alternatives they would choose. Thus hypothetical choice intentions were elicited in a given imagined situation. This was usually repeated with all $n(n-1) / 2$ pairs from a predefined set of $n$ (usually $n=6$ or $n=9$ ) alternatives. Not the alternatives themselves, but symbolic representations of-with only few exceptionsactually existing alternatives were used in the experiments.

In order to account for the fact that alternatives may be represented in different ways, and that choice processes may depend on differences in representation (Payne, 1982), two different methods of presenting alternatives were used. The two alternatives of a pair were presented either by their names (named presentation), by lists of their characteristic features without names 
(described presentation), or both. The features were organized in a dimensional fashion, that is, if price was a dimension the prices of both alternatives were given. In a named condition, for example, the names of two German magazines (e.g., Capital and Spiegel), were presented in one line at the left and right side on a computer screen. In a described condition, the lists of features of the two alternatives were presented next to each other on the left and right side ordered by dimensions. The dimensions' names (e.g. price) were also given (cf. Table 3-1). Most of the experiments used 11 dimensions to describe the alternatives; descriptively informative rather than evaluative dimensions

Table 3-1. Sample Choice Pairs ${ }^{\mathrm{b}}$ in Named and Described Presentation Mode

Journal Subscriptions

Named presentation

Capital

Spiegel

Described presentation

Frequency of publication

Number of articles on science

Monthly

Number of advertisements

120

Price of magazine

6. $\mathrm{DM}^{\mathrm{a}}$

Number of articles on politics

Number of articles on ecology

Number of articles on entertainment

Number of articles on cultural events

Number of pictures

70

Weekly

1

4. $\mathrm{DM}^{\mathrm{a}}$

25

$1 \quad 5$

$5 \quad 3$

$3 \quad 5$

$15 \quad 15$

Number of color pictures

10

Number of pages in magazine

350

3

300

Vacation Areas

Named presentation

Siena, Toscany

Neusiedel am

See, Austria

Described presentation

Vegetation

Lush

Lush

Rainy days

Temperature (C)

Leisure activities

Purchasing power of deutschmark

Abundant

Landscape

1.17

Average

Hilly

Location

Historical sites

Inland

0.86

Popularity

Many

German spoken

Popular

Distance from Heidelberg

Occasionally

$900 \mathrm{~km}$

Mountains

Lakeshore

Few

Very popular

Always

$1700 \mathrm{~km}$

${ }^{\mathrm{a}} \mathrm{DM}=$ deutschmark.

bTranslations. 
were used as well as features, which were only presented in evaluative form in some specific experiments.

Usually, the alternatives were presented on a computer videoscreen. In some experiments described alternatives were presented by an information display board which initially showed only the dimensions' names. The alternatives' features on the dimensions had to be uncovered sequentially by opening small doors.

Various choice domains were used, that is, vacation areas, journal subscriptions, car rentals, university towns, and board games. Most studies used alternatives that were selected from among existing alternatives. In general, sets of alternatives were collected in such a way that decision conflicts were expected for the subjects for at least some pairs of alternatives. In two earlier studies (Aschenbrenner, Albert, \& Schmalhofer, 1984; Schmalhofer, Aschenbrenner, Albert \& Gertzen (submitted), experiment 1), alternatives that were individually elicited from the subjects themselves (according to principles 2 and 3 ) were used. In these experiments the subjects also generated dimensions and the alternatives' features on the dimensions for those alternatives that were used for described presentation. However, systematic differences between experimenter and subject generated alternatives and descriptions were found neither between nor within the subjects. Therefore, because of the subjects' difficulties in constructing alternatives' descriptions, only experimenter-generated alternatives were used in later studies.

In addition to the chosen alternatives, choice latencies and/or the dimensions that were inspected by the subjects were also observed as dependent variables. In some studies the subjects were also instructed to think aloud during their choices.

\section{The Evaluation Tasks}

Some researchers (cf. Six, 1980; Upmeyer, 1982) argue that a high attitudebehavior consistency is trivially found if the situations for attitude assessment and for behavior observation are identical. We disagree with this argument. From a decision-theoretical point of view evaluations have to be assessed with respect to the same situation and objectives as the one in which choices are made, if the former shall predict the latter. For example, the same ingredients of some food may be evaluated completely differently by the same person when the person is on a diet as compared to his regular eating behavior. Therefore, the subjects had to imagine the same choice situation during the evaluation sequence. Consequently, they were asked to evaluate the attractiveness of the alternatives' features with respect to their objectives in the given choice situation. For example, they were asked, "Consider the dimension 'landscape' of your next summer vacation spot. How attractive would be the feature "hilly?" These evaluations were done on 7-point rating scales with the extreme points 1 and 7 being labeled "unattractive" and "very attractive." 
For alternatives used for named presentation the alternatives' features were elicited individually before evaluation by a free-association procedure. Subjects were asked, for example: "If you think about Ajaccio on Corse as a place for your summer vacation, what comes to mind? "The associations and their time sequence were recorded individually. After the subjects' associations had been elicited for all the alternatives that were presented in the choice tasks, the subjects evaluated their associations by the same procedure as used for the features of the described alternatives. The attractiveness ratings were usually elicited by a microcomputer for one feature at a time.

Subjects also evaluated the importance of the dimensions on which the alternatives were described in the described conditions. Subjects were asked, for example: "How important is the following dimension for you when choosing a place for summer vacation: landscape." In earlier experiments a 7-point rating scale and procedure was used. The poles of the rating scale were labeled "unimportant" and "very important". More recent studies used a magnitude estimation procedure for eliciting dimensional importance in which the subjects first rank ordered the dimensions of a choice topic and then indicated how much more important each dimension was as compared to the least important dimension.

Thus the following variables were available for predicting individual choices: feature-attractiveness ratings, dimension-importance values for described alternatives, and association-sequences and their time characteristics for named alternatives. In some studies further variables were assessed for predicting the choices, that is, the inspected dimensions, their order and their number.

One may hypothesize that our variables "attractiveness" and "importance" correspond respectively to Fishbein and Ajzen's predictors "evaluation" and "belief strength." For the first variables the correspondence is self-evident. However, importance and belief strength are not interchangeable expressions. Whereas belief strength is the extent to which a person is convinced that an alternative has a certain feature, the importance of a dimension is assumed to increase with the degree of uncertainty about the features constituting the dimension; that is, importance is considered a measure of the expected amount of choice relevant information that is obtained by learning an alternative's feature on the dimension. Further, attractiveness plays a role similar to that of evaluation in Fishbein and Ajzen's models, whereas the role of importance (in our models) is completely different from that of belief strength.

\section{Criterion-Dependent Choice Models}

The CDC models postulate a sequential evaluation process that results in an intention to choose one of two alternatives when enough evidence has been 
accumulated to be sure that this alternative is better than the other with respect to the situation and objectives. This does not require that all available information has been processed. The moving force of this process is lack of evidence or doubt about the superiority of the currently preferred alternative. Within the framework for cognitive choice processes previously mentioned, the following more specific assumptions are made. They apply to both named and described alternatives.

\section{Information Selection}

It is due to the narrowness of consciousness (Müller \& Pilzecker, 1900, p. 79) that information abut potential choice alternatives are processed sequentially, that is, their cognitively represented features. The features of named alternatives are internal, for example, knowledge or memory based; thus their processing sequence is the sequence of their retrieval. These retrieval sequences may depend not only on the presented names but also on the choice situation, the person's objectives, and so forth. To model this retrieval process is not aspired by the CDC models since models of retrieval from memory are already available (e.g., Raaijmakers \& Shiffrin, 1981; Strube, 1984); they can be adapted to and incorporated into the CDC models (Kühn, Schmalhofer, Albert, \& Aschenbrenner, 1986).

The features of described alternatives are by definition externally presented-in our experiments, in the specific dimensional presentation mode. These features are assumed to be processed in pairs, that is, the features of the two alternatives on one dimension at a time. The processing sequence is assumed to depend on the additionally given dimension names. More precisely, it is assumed that the sequence depends on the importance of the dimensions with respect to the choice problem. At least probabilistically, important dimensions are assumed to be processed earlier than less important dimensions.

Other assumptions about the sequential selection of dimensions of described alternatives may also be introduced into CDC models. For example, one may assume that reading habits influence the sequence; or, if some information is more accessible than other information, salience and availability may play some role.

In light of Fishbein and Ajzen's (1975) discussion of the importance of features as a predictor of behavior, the assumption of processing by importance appears interesting enough for empirical study. Note, however, that we consider importance of dimensions, that is, feature classes, and not importance of individual features as Fishbein and Ajzen do. Furthermore, in the CDC models importance may direct the sequence of information processing; importance is not considered a multiplicative weighting factor for evaluations. 


\section{Evaluation and Aggregation}

The core of every attitude-behavior model consists of assumptions regarding evaluation and aggregation. It is not that the other components of the process are less important for modeling, but they are less typical for the behavior in question. Assumptions about selecting information and terminating information processing may be found in models for almost any behavior (e.g., letter identification or aimed movements). Evaluative and aggregating components may also be found in processes of the latter type. But these components are usually not of the same kind as is typical for attitudebehavior and choice research; namely evaluations of the like-dislike, goodbad, or attractive-unattractive kind.

Such an evaluation is assumed to take place sequentially dependent on the processed features. Every new feature or feature pair that is processed is assumed to be evaluated, and these evaluations are assumed to be accumulated. Evaluations may be absolute or comparative depending on whether an individual feature (named presentation) or a feature pair (dimensionally described presentation) is processed. It is not assumed that the resulting evaluation depends solely on the processed feature or features as a somewhat stable value or association. Rather, evaluations may vary with the respective situation and the subject's objectives. To our knowledge such an evaluation process has not yet been satisfactorily modeled. Which is why we inquire about the evaluations and stress the identity of situations for eliciting choices and evaluations.

Each evaluation is assumed to be aggregated to the results of prior evaluations during the choice process. Depending on which alternative is favored by an evaluation, the aggregated evaluation is changed toward that alternative. Nevertheless, the aggregated evaluation may still favor the other alternative. If the currently evaluated features do not favor any alternative, the aggregated evaluation remains unchanged in that processing step. If the person has no bias for one of the alternatives (e.g., left or right bias) the process begins with a neutral evaluation.

\section{Termination and Choice}

Sequential information selection, evaluation, and aggregation are assumed to stop when the aggregated evaluation reaches or exceeds one of two critical values, one for each alternative. The alternative whose critical value has been reached is chosen. The size of the critical value corresponds to the amount of evidence or accumulated attractiveness difference which is necessary in order to be certain that one alternative is the better one, although some information has not been processed. We still assume that the size of the critical value does not depend on the particular choice alternatives but on characteristics of the situation, the subject, or other constraints such as choice quality, time pressure, and so forth. Although effects of such parameters on 
the direction of changes of the critical value are easy to imagine, a precise formulation of the dependence requires further research.

In this context behavioral choice means that at the time of terminating the process, and under the given circumstances, a person would intend to realize the chosen alternative. However, it may happen that the (internally or externally) available information is not sufficient for exceeding one or the other critical value. In particular this may occur for similar alternatives or for high critical values. In this case several alternative possibilities are available, for example, searching for further information, repeated processing of the information using other heuristics, choosing at random, or concluding that the critical value was too high and deciding on the basis of a smaller evaluative difference. The latter rule was applied to our data because it seemed to be the most rational and simple in a forced-choice situation, and it coincides with the evidence interpretation of the critical values: if all information has been processed, instead of an evidence criterion the "exact" attractiveness difference may be used for making a decision.

Summing up our main theoretical assumptions in a more formal way, Figure 3-1(a) presents a flow diagram of the CDC models for named and described alternatives, Figure 3-1(b) illustrates the process model for the choice between dimensionally described alternatives, and Figure 3-1(c) is an illustration of the assumed process for the choice between named alternatives.

The common characteristics of the CDC models are sequentialinformation processing and criterion-dependent termination of the choice process. Specific characteristics, for example, the way in which features are evaluated and aggregated or the number of features (one or two) that are processed in one step, may differ between the models. Furthermore, the sequence of processing features may be constant or may vary probabilistically. These and other differences between the specific models are all compatible with these general assumptions. The main reasons for designing this class of models are:

1. The CDC models incorporate a number of well-known choice heuristics (cf. Svenson, 1979) and allow the development of stochastic versions. For example, with an ordinal-scale level for the evaluations and with features being processed in pairs, the lexicographic rule is obtained if the critical value is set to one; with large critical value the majority rule is approached.

2. The assumption of criterion dependence continues a tradition of decision models (Audley, 1963; Audley \& Pike, 1965; Bower, 1959; Estes, 1959) that have been discussed and successfully applied in perception (e.g., for discrimination decisions; Link, 1978; Link \& Heath, 1975) and memory research (e.g., for recognition decisions; Ratcliff, 1978), since the beginning of the 1960s; in probability theory related models (e.g., random walk models, sequential sampling models; see Feller, 1957; Wald 1950) are even older. In contrast to these models is the CDC models' assumption that 


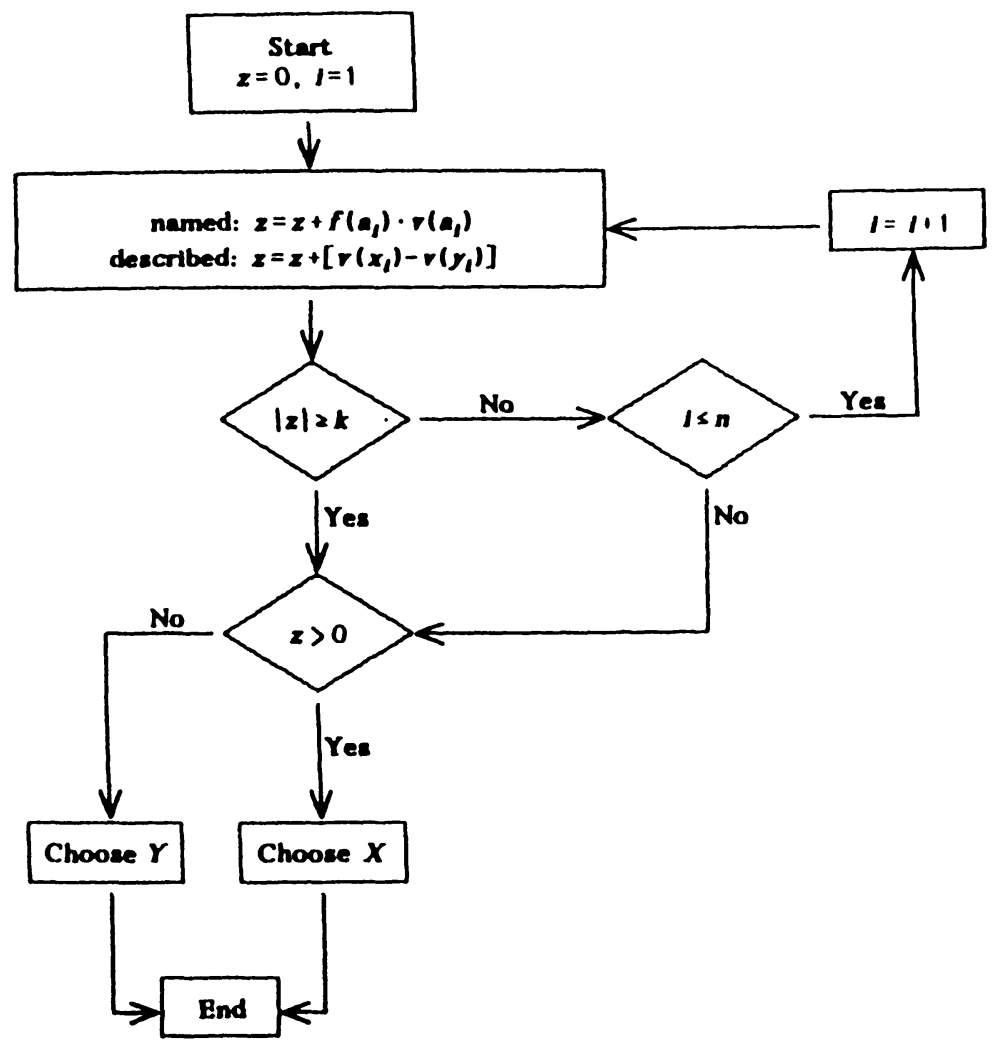

(a)

Figure 3-1(a) Flow diagram of the CDC model for named and described alternatives. $Z$ is the counter, $k$ is the critical value, $i$ is the number of the processing step. For named alternatives: $a_{1}, a_{2}, \ldots, a_{i}, \ldots, a_{n}$ is a sequence of features. $f\left(a_{i}\right)$ is 1 if $a_{i}$ is a feature of alternative $X$, and $-l$ if $a_{i}$ is a feature of alternative $Y$. For described alternatives: $x_{i}$ and $y_{i}$ are the features of $X$ and $Y$ on the $i$ th dimension. $v($.$) is a feature's$ evaluation with values between -3 and +3 . (b) Illustrative example of a CDC choice process between two described alternatives. (c) Illustrative example of a CDC choice process between two named alternatives.

every feature of each alternative or at least its evaluation is processed only once, that is, no replacement is assumed. This complicates the formal mathematical analysis of the models.

Recently, the tradition of criterion dependence of decisions has been continued by Busemeyer (1982, 1985), Wallsten (1980), and Wallsten and Barton (1982) for described stimuli. Wallsten predicted probabilistic inference decisions with this kind of model; Busemeyer's subjects had to choose between a reliable and a risky alternative. The empirical findings of these studies are encouraging; thus-together with the previously mentioned incorporation of well-known choice heuristics-the criterion 


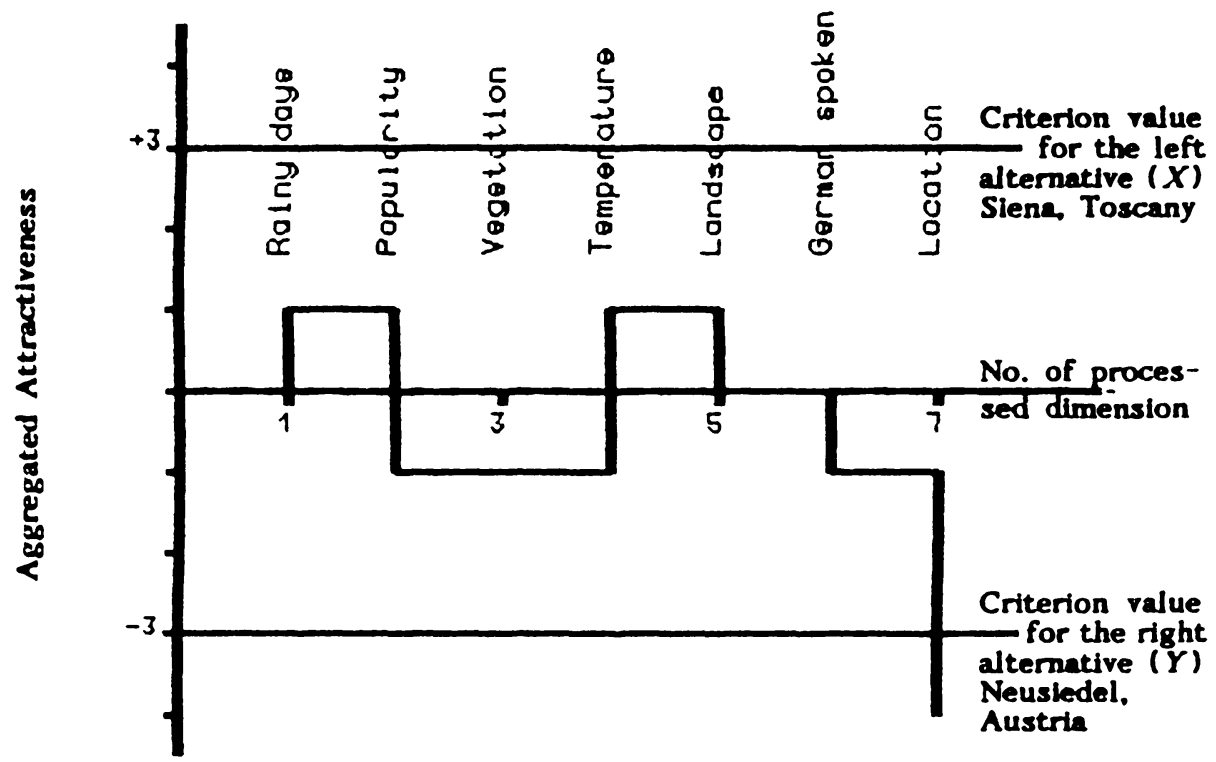

\begin{tabular}{|c|c|c|c|c|c|c|c|}
\hline$\left[r\left(x_{1}\right)-r\left(y_{1}\right)\right]$ & +1 & -1 & -1 & +1 & 0 & -1 & -4 \\
\hline$r\left(x_{1}\right)-v\left(y_{1}\right)$ & +1 & -2 & 0 & +2 & -1 & -1 & -3 \\
\hline$r\left(x_{1}\right)$ & -2 & +1 & +2 & -1 & 0 & -1 & -3 \\
\hline$r\left(y_{1}\right)$ & -3 & +3 & +2 & -3 & +1 & 0 & 0 \\
\hline$x_{1}$ & 15 & Popular & Lush & 29 & Hilly & $\begin{array}{l}\text { Occa- } \\
\text { sionally }\end{array}$ & Inland \\
\hline$y_{1}$ & 2 & $\begin{array}{l}\text { Very } \\
\text { popular }\end{array}$ & Lush & 20 & Plain & Always & Lakeshore \\
\hline
\end{tabular}

(b)

Figure 3-1. (Continued)

dependence principle may help to unify theorizing in choice and decision research, as well as attitude-behavior research.

3. The CDC models allow for systematic (through experimental manipulation) as well as unsystematic (through probabilistic fluctuation) variation of choices and choice latencies. Such variation may have the following causes: systematic or unsystematic variation of the sequence of processing features, the critical value, the attractiveness values, or the starting value for the aggregation of evaluations.

4. As a consequence of systematic variations the CDC models allow for modeling different effort-quality trade-offs by manipulating the size of the critical values (Schmalhofer, 1987; Schmalhofer \& Saffrich, 1984). 


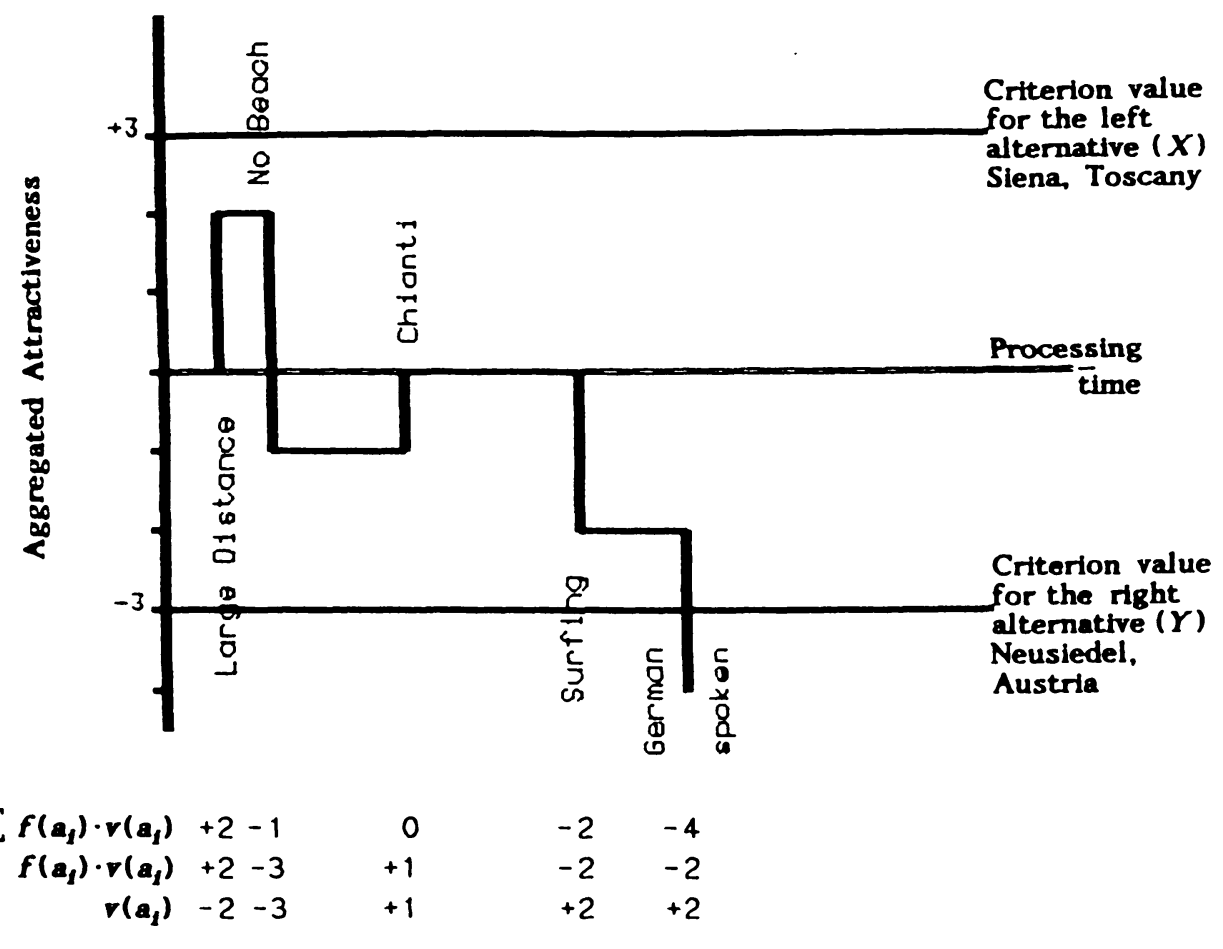

(c)

Figure 3-1. (Continued)

5. The CDC models capture the well-known context dependency of choices (Payne, 1982), that is, the dependence of the chosen alternative on the context of the other alternatives. This effect is responsible for not allowing simple scaling of choice alternatives.

6. The CDC models describe the oscillation between choice alternatives known from experience in which one's temporary preference vascillates from one alternative to the other during the choice process. Likewise, the CDC models may describe the unequivocal preference of one alternative following a relatively fast choice process.

7. The CDC models are in line with the attitude-behavior models in the Fishbein and Ajzen tradition; although we would like to assert that at least for binary choice the cognitive processes are described more adequately by the CDC models, since they modify and extend the processing part of Fishbein and Ajzen's theory.

By making the choice dependent on reaching a criterion (instead of summing up $n$ evaluations), and by introducing assumptions about the sequence 
of information processing, Fishbein and Ajzen's problem of using exactly those beliefs that are salient is solved.

\section{Empirical Results}

Even though the discussed aspects seem to be desirable theoretical reasons for designing CDC models, there is still a need to provide empirical support for them. The following studies will be organized by the three components of the CDC models: information selection, evaluation and aggregation, and termination by a choice.

Implications of specific CDC models were empirically tested in a series of experiments and quasiexperiments. Usually each investigation focused on specific aspects of some CDC model. Therefore, results from various studies will be organized in such a way that they refer to the previously mentioned three components of the CDC models.

Prior to this presentation, we would like to say a few words about global measures of model fit, such as number of correct predictions. A good overall fit provides only restricted evidence for the validity of a model (cf. the critical comments previously discussed regarding Fishbein and Ajzen's empirical procedures). Good overall fit is, however, a high incentive for more specific tests of a model. Table 3-2 displays the relative frequencies of correctly predicted choices that were obtained in various studies which used named as well as described presentation of alternatives. In each case, the choices were predicted individually by the respective CDC model variant and by means of the following parameters: attractiveness judgments, sequences of associated features to named alternatives, and importance judgments or observed sequences of inspected dimensions for described alternatives. The critical value was estimated as a free parameter from the choice data individually for each subject and choice topic. That is, one critical value was used for all

Table 3-2. Relative Frequencies of Correctly Predicted Choices

\begin{tabular}{lcc}
\hline & $\begin{array}{c}\text { Named } \\
\text { Alternatives }\end{array}$ & $\begin{array}{c}\text { Described } \\
\text { Alternatives }\end{array}$ \\
\hline Schmalhofer et al. (1986) & - & $.80^{\mathrm{a}}$ \\
& - & $.83^{\mathrm{b}}$ \\
Schmalhofer et al. (submitted) & $.73(.65)^{\mathrm{d}}$ & $.83(.78)$ \\
Experiment 1 & - & $.80^{\mathrm{c}}(.74)$ \\
Experiment 2 & $.78^{\mathrm{c}}(.76)$ & - \\
Experiment 3 &
\end{tabular}

${ }^{\text {a }}$ Predicted by the most extreme countervalue without a critical value.

${ }^{b}$ Predicted by sign of the last countervalue without a critical value.

${ }^{c}$ Averaged over two salience conditions.

${ }^{d}$ In parantheses the frequencies with critical values estimated independently. 
choices of a subject from a set of alternatives. In some cases, the critical value was estimated to maximize the number of correct predictions for a subject. In other cases, only part of the choices was used to estimate the critical value which was then used to predict the other choices.

Overall, the model fit appears satisfactory, although it is generally somewhat lower for named alternatives than for described ones. Also, as expected, the fit is somewhat lower but still satisfying if the critical value is estimated independently. It should be noted, however, that model fits being accomplished by the traditional additive model (i.e., by adding the attractiveness values of all features of each alternative and choosing the alternative with the higher sum of values) are in the same range (see the section on comparison with other models). Thus these global results not only suggest but also require more specific tests that will be discussed in the following sections.

\section{Information Selection}

Information concerning choice alternatives may be represented internally in the memory of a person (when the name of an alternative is presented) or externally (when a description of an alternative is given) or both (in a shop products do often have their names as well as some descriptive information printed on the package). In our studies, alternatives were presented either by name only or by lists of features organized by dimensions without names, or both.

For named alternatives we assume that individual features of the alternatives are processed in their order of recall, that is, in the order in which they come to the subject's mind. For described alternatives we assume that features are processed in pairs in the order of the dimensions' importance. These assumptions were supported by think-aloud protocols from 20 subjects who had to choose between named as well as described alternatives in a balanced order (Schmalhofer \& Schäfer, 1986). Examples of protocol excerpts from vacation choices indicating single feature processing are: "12 rainy days is rather bad, isn't it?"; "very hot weather." Examples indicating processing in pairs are: "temperature is rather cool there, hot here"; "well, I'd prefer warm to hot and humid weather." As expected, single-feature processing occurred significantly more often with choices between named alternatives, whereas pairwise processing prevailed with dimensionally described alternatives (see Table 3-3).

For further testing of assumptions about processing order, twenty-four subjects had to choose among the same alternatives presented once by name and once by description. Also, both free associations to the alternatives' names and judgments of the importance of the dimensions used for describing the alternatives were elicited. Although we do not consider an overall model fit very informative on an absolute level, it should be sensitive as a relative measure for comparing different processing assumptions. Thus we predicted the choices under both presentations by both processing assumptions. As expected, the choices between named alternatives were better pre- 
Table 3-3. Frequency of Occurrence of Utterances Referring to Individual Features and of Utterances Referring to Pairs of Features on a Dimension (e.g., Comparisons) in Think Aloud Protocols

\begin{tabular}{clcc}
\hline & & \multicolumn{2}{c}{ Choice Pair } \\
\cline { 3 - 4 } Sequence & Object & Named & Described \\
\hline \multirow{2}{*}{ Named-described choices } & & $n=160$ & $n=577$ \\
& Feature & $43.65 \%$ & $29.64 \%$ \\
& Dimension & $8.10 \%$ & $31.54 \%$ \\
Described-named choices & & $n=212$ & $n=646$ \\
& & $44.34 \%$ & $25.55 \%$ \\
& Feature & $8.02 \%$ & $39.02 \%$ \\
\hline
\end{tabular}

From Schmalhofer \& Schăfer (1986), with permission.

dicted when the processing orders were assumed to be identical to the subjects' retrieval sequences than when the processing of feature pairs was assumed to be in the order of dimensional importance. For described alternatives, the opposite result was obtained (see Table 3-4).

The results reported thus far were obtained under the assumption of constant processing orders within subjects. For described alternatives it was assumed that a subject processed the feature pairs in the same order for all pairs of alternatives. Only the number of feature pairs could vary between choice pairs because the attractiveness differences on the dimensions determine how fast one of the criteria is reached. Similarly, the same feature retrieval order that was obtained for a named alternative by free association was assumed to occur every time when that alternative was presented in a choice pair independently of the other alternative in the pair.

One reason for questioning this simplifying assumption is that subjects do not always choose the same alternative when presented with the same pair of alternatives repeatedly (e.g., Petrusic \& Jamieson, 1978). This was also observed in our studies. For example, when subjects had to choose between the

Table 3-4. Percentage of Correct Predictions of Choices Between Named and Described Alternatives by Either CDC Model

\begin{tabular}{lcc}
\hline & \multicolumn{2}{c}{ Choice Between } \\
\cline { 2 - 3 } \multicolumn{1}{c}{ CDC Models } & Named Alternatives & Described Alternatives \\
\hline Named alternatives & $73 \%$ & $68 \%$ \\
Described alternatives & $69 \%$ & $83 \%$ \\
\hline
\end{tabular}

From Schmalhofer, Aschenbrenner, Albert, \& Gertzen (submitted). 
same alternatives twice with a retest interval of about one week, the average percentage of identical choices over two conditions in the two sessions was $0.86 \%$ for named and $0.85 \%$ for described alternatives (Schmalhofer, Aschenbrenner, Albert, \& Gertzen, submitted). In light of this reliability of choices, the average goodness of fit of about $80 \%$ correct predictions previously reported can be regarded as very good.

The CDC models can account for this fluctuation in choices in a number of ways. The attractiveness evaluations of the features may fluctuate (Thurstone, 1927), the critical value may fluctuate, and finally the order of processing may vary. The latter aspect of varying processing sequences is new and distinguishes the CDC models from other choice models. Since the CDC models assume that only parts of the available information are processed until a criterion is reached, different processing orders may result in the processing of different information about the alternatives and may thus yield different choices when the same alternatives are presented twice. These fluctuations may also be responsible for less than perfect predictions.

The aspect of different processing sequences was investigated intensively in a number of experiments. One experiment (Aschenbrenner, Albert, \& Schmalhofer, 1984) tested the assumption of varying processing orders against the traditional value-fluctuation assumption for described alternatives. Four topic fields (vacation areas, car rentals, journal subscriptions, and university towns) were used. Two of these were used for named and two for described presentation in a balanced design. In order to assess relatively stable estimates of individual choice fluctuations (i.e., choice probabilities), 36 choice pairs (all pairs of 9 alternatives) per topic field were presented to 8 subjects repeatedly over 20 sessions with weekly intervals. Feature evaluations and importance judgments were also elicited in every session.

All subjects had some choice pairs (about $1 / 3$ to $1 / 2$ ) for which their choices varied. Also the resulting choice probabilities were inconsistent with more simple stochastic choice models such as the Bradley-Terry-Luce model (Luce, 1959), which assumes choice probabilities to be a simple function of the alternatives' overall values. One version of a CDC model assumes a constant processing order for all sessions, but varying feature evaluations (Thurstone, 1927). The actual variations of the evaluations over sessions were used to estimate this fluctuation. Another version of the CDC model assumes constant evaluations-for each feature its average evaluation over the 20 sessions was used-but the sequence of processing dimensions was assumed to vary probabilistically (Albert, Schmalhofer, \& Aschenbrenner, 1983). The probability for selecting an initial dimension or the next dimension in a choice was assumed to be a certain monotone function of the dimensions' importance (see the scale considerations afterwards). The critical values were estimated separately for each subject, topic field, and CDC model so that the fit was maximized.

Both CDC models predicted some choice variation for all subjects which covaried with the observed variation. Model fit was determined by calculat- 
ing the average quadratic deviation between predicted choice probabilities and observed choice frequencies over all pairs from a topic field for each subject separately. The predictions derived from the assumption of varying processing sequences fitted the subjects' choices more accurately $(p<0.002)$ than the predictions derived from the value-fluctuation assumption in 14 out of 16 cases ( 8 subjects $\times 2$ topic fields).

Thus the order of processing information and its variation appears to play a substantial role in choice processes. Consequently, this subprocess was analyzed in more detail in further experiments.

In order to observe the selection of dimensions and the fluctuation directly, experiments were run in which dimensions' names were presented while keeping the alternatives' features on the dimensions covered. Subjects had to select a dimension when they wanted to see the alternatives' features on that dimension. This procedure allows for direct observation of the subject's process of selecting information so that it is not necessary to rely on overall model fit, since assumptions about the selection process can be tested directly. The assumption that the selection process may be described probabilistically by Luce's choice axiom was investigated; that is, that the probability $p_{i J}$ for choosing a dimension $i$ from the choice set $J$ of dimensions is

$$
p_{i J}=\frac{s_{i}}{\sum_{j \in J} s_{j}} .
$$

The choice axiom has the important property that the dimension parameters $s_{i}$ are invariant under different sets of dimensions. Naturally the choice set should consist of the dimensions $J$ not yet inspected, since the multiple inspection of the same dimension may be considered as uneconomical. In this case the choice axiom provides a very parsimonious principle of selecting dimensions, since the dimensions' parameters $s_{i}$ remain constant for the successively smaller sets of dimensions. If the choice axiom holds (which is usually not the case for choices between multidimensional alternatives; Luce, 1977), the objects in question-in our case the dimensionsmay be ordered along a continuum. According to the previously described considerations the continuum should reflect the dimensions' importance. Direct scaling of the dimensions' importance by an adequate judgment procedure should result in importance values having a monotone relationship with the indirectly obtained scale values of the choice axiom, provided that the choice axiom adequately represents the selection process, and that importance judgments obtained by the direct-scaling procedure depend on the same underlying variable.

In order to test these assumptions Aschenbrenner, Böckenholt, Albert, and Schmalhofer (1986) reanalyzed data from two experiments. In both experiments 15 binary choice tasks consisted of choosing a 1-year subscription to a news magazine. While a display board always revealed the names of the 11.dimensions (see Table 3-1) by which the alternatives were described, the 
features of a choice pair on the dimensions had to be successively requested by opening small doors until the subject wanted to make a choice. Additionally, the subjects judged the dimensions' importance on a 7-point rating scale.

The $s_{i}$ parameters were estimated individually from each subjects' 15 orders of inspecting dimensions by an iterative maximum likelihood algorithm described by van Putten (1982). Two likelihood-ratio tests were performed. First, the choice axiom with the estimated parameters (the fitted model) against the unrestricted model which reproduces the data perfectly was tested. Second, an even more restricted model assuming equal $s_{i}$ values for all dimensions against the fitted choice model was tested. The latter assumption of equal $s_{i}$ values for the choice model was rejected for 46 out of 49 subjects while the fitted model was accepted for all but 1 subject. This demonstrates that the assumptions of the choice axiom and of the fitted parameters are meaningful since both could have been violated.

With respect to the relation between judged importance and importance inferred from the choice axiom, the results were less conclusive. Although we obtained positive correlations between the $s_{i}$ values and the importance ratings for most of the subjects in both experiments, they were not high enough for the consideration of both variables as indicators of the same underlying variable or for considering one variable as some form of validation for the other. An underlying cause might have been that a rating procedure for the elicitation of importance judgments is far from being optimal when the required ratio-scale level is considered.

Thus, another study was performed (Aschenbrenner, Böckenholt, et al., 1986, experiment 3) using magnitude estimation for eliciting importance judgments. Two groups with different payment of 20 subjects each had to choose between pairs of new board games described on 8 dimensions. This experiment used a pair comparison procedure for observing dimensional selection, that is, only two dimensions at at time were offered. After inspecting the alternatives' features on one of the two dimensions, the subjects were allowed to choose one of the two alternatives or to select another dimension from a new pair of dimensions. They had to make a choice by the time they came to the last dimension. Choosing between pairs of alternatives was continued until each of the 28 possible pairs of dimensions had been offered at least 20 times in order to gather sufficiently stable estimates of the dimensions' selection probabilities.

After the choices the dimensions' importance was evaluated by applying a direct scaling procedure, namely a magnitude estimation procedure. Subjects first rank ordered the eight dimensions and then had to judge how much more important each dimension was as compared to the least important dimension.

The $s_{i}$ values were estimated individually from the pairwise dimensional selection data by maximum likelihood. The probabilities of the observed selection frequencies under the assumption of the choice axiom and the es- 
timated parameters were calculated. These probabilities resembled the expected values very well, thus indicating the appropriateness of the choice axiom and the $s_{i}$ parameters.

The individual subjects' rank correlations between the estimated $s_{i}$ values and the judged importance values $w_{i}$ were generally high, with medians of 0.88 and 0.94 in the two groups of subjects. If both $s_{i}$ and $w_{i}$ values are measures of the same underlying importance continuum and the assumptions about their scale levels are fulfilled, the logarithms of the values of the two scales should be linearly related. Usually, the $s_{i}$ values are considered to have ratio-scale level, whereas magnitude estimates are assumed to have a log-interval-scale level (e.g., Krantz, Luce, Suppes, \& Tversky, 1971, pp. 164166). Indeed we found the product-moment correlations between $\log \left(s_{i}\right)$ and $\log \left(w_{i}\right)$ generally higher than those between $s_{i}$ and $w_{i}, \log \left(s_{i}\right)$ and $w_{i}$, and $s_{i}$ and $\log \left(w_{i}\right)$; thus supporting the hypothesis. Examples for the $\log \left(s_{i}\right)$ and $\log$ $\left(w_{i}\right)$ relationship are presented in Figure 3-2.

Taken together, since the widely recognized assumption of sequential information processing was supported by global model tests, we presupposed sequential information processing for dimensionally described alternatives in order to make the processing observable with the aid of appropriate techniques. Thus data supporting two assumptions could be collected: (1) the variation in information selection can be described probabilistically in agreement with Luce's choice axiom, and (2) the estimated-scale values of the dimensions are closely related to the directly elicited subjective importances of the same dimensions. Both the scale values and importance judgments may be considered as indicators of the same latent variable. In our interpretation, the latent variable that orders the dimensions determines the subjectively expected amount of information about the alternatives and their features with respect to the specific choice task. Thus we are now able to investigate which factors determine the ordering of the dimension along the latent variable in a specific choice situation for an individual with certain objectives, values, and knowledge.

Unfortunately, we do not have a technique for observing information retrieval from memory for named alternatives in the same direct manner as for described alternatives. However, there are some indications that the sequential process of information retrieval can also be described approximately in probabilistic ways (e.g., Albert, 1968; Shiffrin, 1970), though only for features and not for dimensions.

In addition, another difference must be considered. For described alternatives we postulated that each dimension is processed only once; thus we assume probabilistic selection without replacement. However, for named alternatives we need to assume probabilistic retrieval of features with at least partial replacement, as shown in the previously established models of recall and continuous association in their comparison with models without replacement (Albert \& Schulz, 1976). By replacement of features it is meant that the choice set in memory may remain the same during the choice pro- 

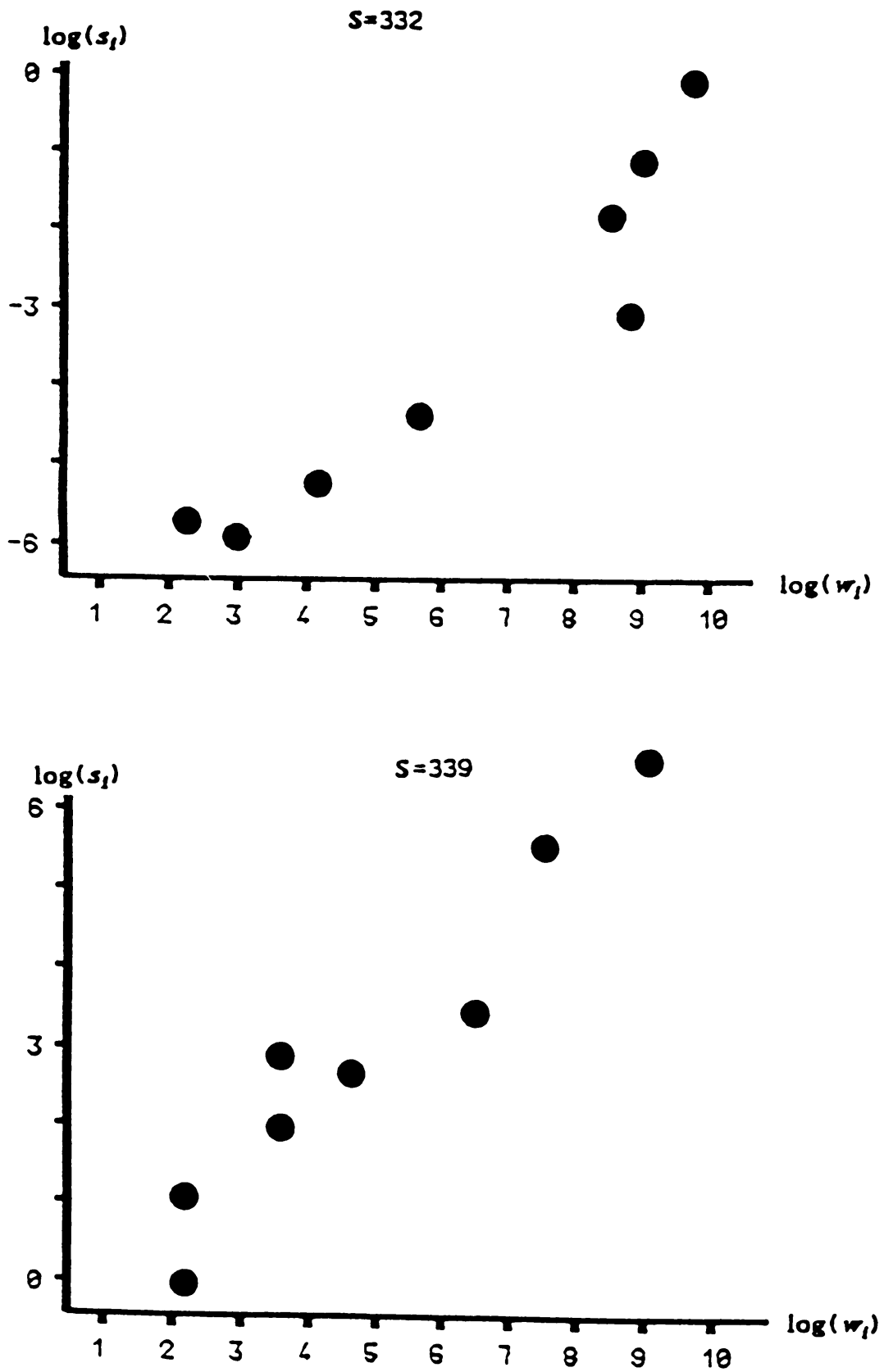

Figure 3-2. Sample plots of the $\log \left(s_{i}\right)-\log \left(w_{i}\right)$ relation for four subjects of experiment 3 in Aschenbrenner, Böckenholt, Albert, \& Schmalhofer (1986), with permission. 

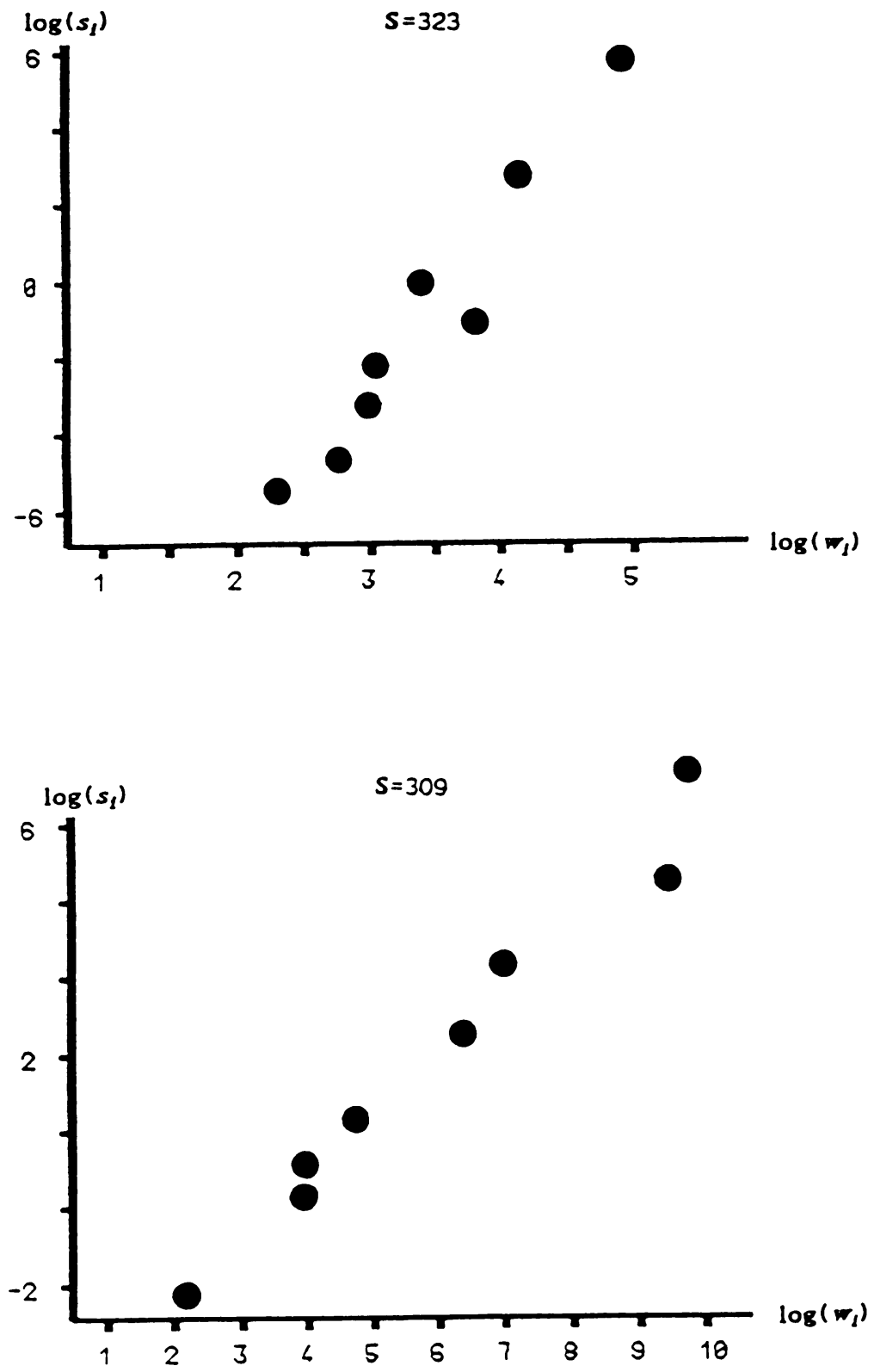

Figure 3-2. (Continued) 
cess and that an already processed feature may be selected again. Although this may be time-consuming it does not necessarily influence the choice, since reprocessing of a feature does not necessarily mean reevaluation.

Finally, there is the problem that association sequences to pairs of alternatives (during choice) are inferred from association sequences to single alternatives (during elicitation). Thus the temporal order of the retrieved features of an alternative should be independent of whether or not other features of a different alternative are in the retrieval set. Support for this assumption is provided by Albert and Schulz (1981), who demonstrated that the choice axiom still holds for simultaneous reproduction from two or more lists that were separately learned.

Nevertheless, the influence of other factors, especially semantic ones, should not be disregarded in memory retrieval. Therefore, the retrieval of features does not only depend on the corresponding memory or retrieval strength of the feature, but also on the specific features that have been retrieved before. It should also be noted that such semantic factors can play a role in addition to their importance in the selection of externally presented dimension names. This effect might not have been discovered in our data because we tried to use semantically and functionally unrelated dimensions for describing the alternatives. Thus other factors may influence the selection sequence in addition to the choice axiom, which may be considered as a good first approximation.

\section{Evaluation and Aggregation}

The general model and the global model tests (Table 3-2) that were previously mentioned include assumptions about the evaluation and aggregation for both named and described alternatives: The often used differentiation between cardinal and ordinal values and comparisons is made (Svenson, 1979). In either case, a counter or summation operation-as in many choice heuristics-is assumed as an aggregation rule. The cardinal models require a higher (interval) scale level than the ordinal model variants for the cognitively aggregated as well as for the separately assessed attractiveness values. Whether this scale level can be obtained for the attractiveness ratings is doubtful, as demonstrated by results obtained with Orth's (1979, 1982) method (Laier, Albert, Schmalhofer, \& Aschenbrenner, 1986). On the other hand, the percentages of correctly predicted choices in our studies were usually higher for cardinal than for ordinal models. This also holds for the modified version with stochastic dimensional selection. Therefore, it can be assumed that the attractiveness ratings contain more than ordinal information.

For dimensionally described alternatives we may again try to take advantage of the possibility of observing dimensional selection for directly testing hypotheses concerning the evaluation and aggregation component. In an unpublished experiment, subjects first rank ordered the dimensions accord- 
ing to importance and rated the features' attractiveness values. One to two days later we presented successively each dimension's name together with the features of two alternatives in the order of judged importance. After each presented dimension the subject had to make a tentative choice specifying which of the alternatives he would now choose.

Thirteen subjects participated in this experiment. The choice areas were vacation places in August and news magazine subscriptions. The subjects had to choose between 6 alternatives, that is, 15 choice pairs, for each choice area. By aggregating the rated attractiveness values in the same order as the features on the 11 dimensions were presented to the subjects, we were able to predict about $85 \%$ of the tentative choices correctly.

Of special interest are those cases where the subjects changed their tentative choice during a choice process. This occurred in 214 out of the 1949 observed tentative choices. If the modeled variations of the counter value correspond to the subjective oscillations between the alternatives, then tentative choice changing should occur only if the counter changes correspondingly. The last two rows of Table 3-5 (a) confirm this correspondence (the 41 choice shifts that were accompanied by no change in the counter value may be due to the subjects discriminating finer than the 7-point rating scale allowed).

However, a similar result would be expected if the subjects would not aggregate evaluation differences over dimensions at all but choose only on the basis of the last dimension they see. The first two rows of Table 3-5 (a)

Table 3-5. (a) Changes in the Counter and Shifts in Tentative Choices;

(b) Attractiveness Differences and Preference Judgments

\begin{tabular}{|c|c|c|c|c|}
\hline \multirow{2}{*}{\multicolumn{2}{|c|}{$\begin{array}{c}\text { Tentative } \\
\text { Choice }^{\mathrm{a}}\end{array}$}} & \multicolumn{3}{|c|}{ Counter Changes Toward } \\
\hline & & \multirow{2}{*}{$\frac{\text { No Change }}{324}$} & \multirow{2}{*}{$\frac{X}{368}$} & \multirow{2}{*}{$\frac{Y}{239}$} \\
\hline Remains & $X$ & & & \\
\hline & $Y$ & 312 & 198 & 294 \\
\hline Changes & $X$ to $Y$ & 24 & 5 & 70 \\
\hline from & $Y$ to $X$ & 17 & 94 & 4 \\
\hline
\end{tabular}

(a)

\begin{tabular}{|c|c|c|c|c|}
\hline \multirow{2}{*}{\multicolumn{2}{|c|}{$\begin{array}{l}\text { Alternative } \\
\text { Judged to } \\
\text { Be Better }\end{array}$}} & \multicolumn{3}{|c|}{ Difference on Dimension Toward } \\
\hline & & \multirow{2}{*}{$\frac{\text { No Difference }}{195}$} & \multirow{2}{*}{$\frac{X}{298}$} & \multirow{2}{*}{$\begin{array}{l}Y \\
37\end{array}$} \\
\hline Remains & $X$ & & & \\
\hline & $Y$ & 204 & 31 & 279 \\
\hline Changes & $X$ to $Y$ & 151 & 22 & 283 \\
\hline from & $Y$ to $X$ & 121 & 294 & 24 \\
\hline
\end{tabular}

(b)

${ }^{a}$ Missing values are due to simultaneous pressing of two response buttons. 
show that this is not the case. There are many cases in which the currently presented dimension favored the alternative that is not preferred. Actually, there are so many such cases (437) that one might develop the opposite suspicion that the subjects did not care about the given information but simply stuck to their choices. To rule out this possibility a third session one or two days later was similarly run. In this session the subjects were instructed to indicate which alternative was preferred on the currently presented dimension. The results in Table 3-5 (b) show a completely different pattern, thus ruling out both alternative explanations.

Again, there are also many changes in the judgments without corresponding attractiveness differences. These may be explained by the comparison procedure used, in which subjects were able to discriminate between the features' attractiveness when they were presented together. However, due to the small number of categories of the absolute judgment scale, they assigned equal values to these features.

Thus the evaluation differences obtained by the comparative judgments do not always coincide with the evaluation differences calculated from the absolute judgments, that is, attractiveness ratings. This observation is again related to the question concerning the reliability of the ratings and the fluctuation of the implicit attractiveness evaluations.

The rating data of Aschenbrenner, Albert, \& Schmalhofer (1984) were reanalyzed with respect to the consistency of the subjects' attractiveness judgments. The product-moment correlations for these data are presented in Table 3-6 for intervals between 1 and 19 weeks.

The results are in the usual range for rating data; the consistencies increase with shorter intervals as expected. In most of our studies the time intervals were considerably shorter than one week, since the ratings and the choices were often collected in the same experimental session. Although we may consider the consistency of a subject to be higher within one meeting than after a one-week interval, we cannot rule out the possibility that values still fluctuate in this situation. It should be possible to recognize the influence of such value fluctuations if the sequence of processed information is known. Therefore we looked for differences between the $80 \%$ correctly predicted and the $20 \%$ incorrectly predicted choices of an experiment in which the subjects had to uncover the information about the alternatives sequentially (Schmalhofer, Albert, Aschenbrenner, \& Gertzen, 1986).

The incorrect choice predictions do not necessarily indicate a structural difference between the model predictions and the observed data. Instead, the deviations might be explained by inaccuracies in the empirically assessed attractiveness ratings. While the average attractiveness difference for the correctly predicted choices is clearly in favor of the chosen alternative (1.09), the respective differences favor the rejected alternatives only slightly (mean $=$ 0.40 ) for incorrect predictions. If the attractiveness evaluations determine the choice, the preferred alternative should receive higher mean attractiveness evaluations than the rejected one. If this statement is valid for the choices not 


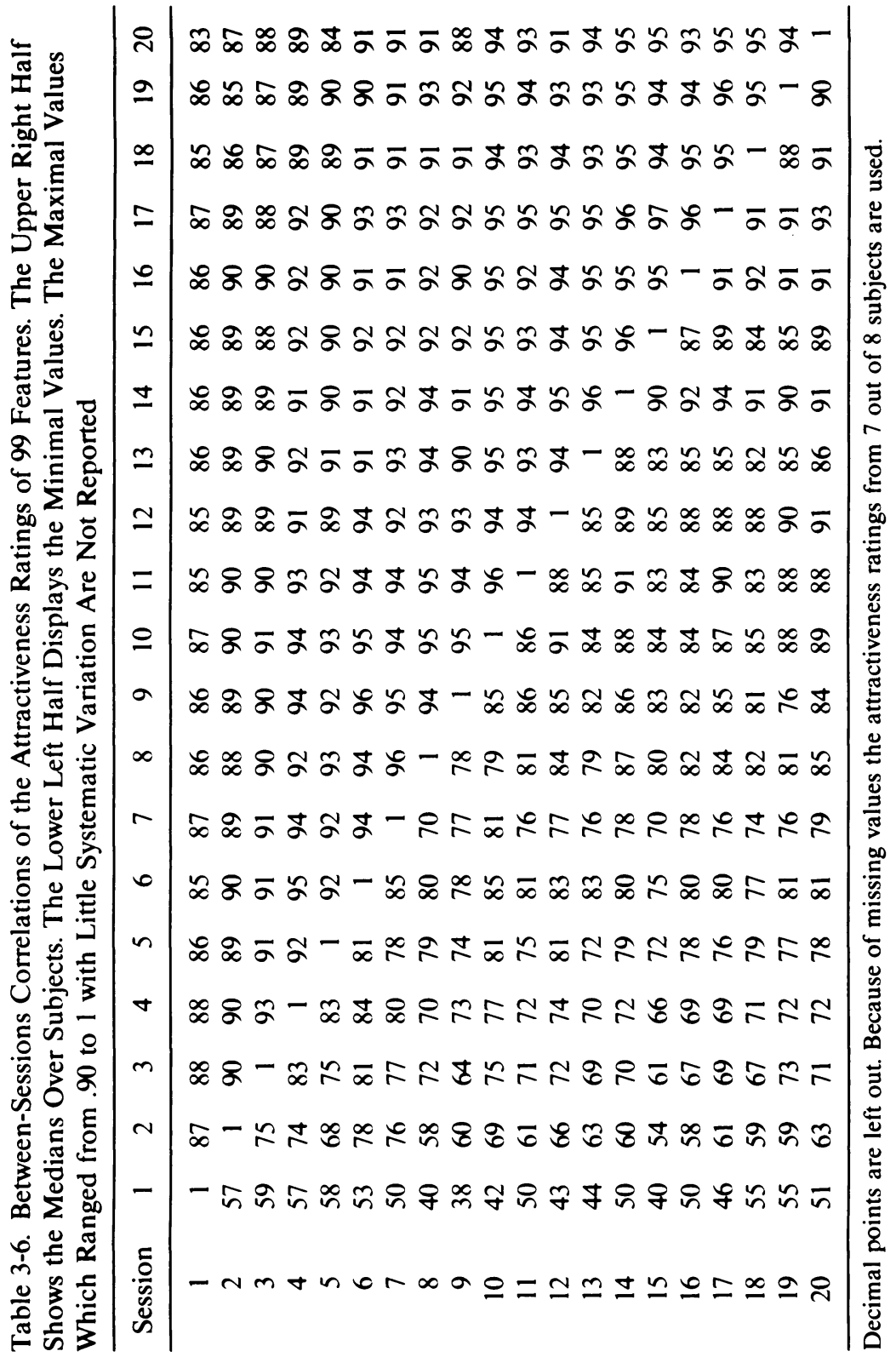


predicted by the model, and if attractiveness evaluations also determine the ratings, then the attractiveness values obtained by the rating procedure may not be identical to the attractiveness values at the time of the choice. This may lead to incorrectly predicted choices, particularily for choice pairs with small attractiveness differences.

\section{Termination and Choice}

The majority of choice models are based on the assumption of complete or at least constant information processing. Hence these choice rules are not able to predict systematic choice latency differences or differences in the amount of processed information. Some choice heuristics are able to do this, for example, the lexicographic rule, the elimination by aspect rule and the CDC rule. According to the CDC model, the choice process is terminated as soon as the accumulated attractiveness values or value differences reach or exceed a criterion in favor of one of the alternatives. Consequently, the amount of information required for a choice is dependent on the actually occurring attractiveness values or differences, and on the order in which these values or differences occur.

The model predicts the number of processing steps for a single choice of a person with known or estimated processing order, attractiveness values, and critical value. Although the time for each processing step may vary, a positive correlation is to be expected between the predicted numbers of processing steps and the observed choice latencies. However, the size of the positive correlation cannot be specified, since individual latency times are usually not very reliable. The correlation of choice latencies with a test-retest interval of one week has been found to be .26 and .30 for choices between named alternatives, and .15 and .24 for choices between described alternatives (Schmalhofer et al., submitted). In addition, due to the previously mentioned consistency and estimation problems, the CDC model cannot perfectly predict the number of processing steps even if the model provides a valid representation. However, we may conclude from significantly positive correlations that the CDC model is superior to other models that assume that the amount of information is the same during choices (of the same type and from the same area). Assuming deterministic processing orders we found a correlation of 0.23 (or 0.12 for only those choices that were not used to estimate the critical values) for named alternatives using free-association data. For described alternatives we obtained correlations of $-0.07(-0.09)$ and $0.05(0.14)$ when deterministic orders processing by importance were assumed (Schmalhofer et al., submitted). Both stochastic models, the stochastic dimension selection, and the value fluctuation model yielded higher correlations between the predicted number of processing steps and choice latencies. Out of 32 taucorrelations 29 were positive; the medians were 0.49 (subject generated alternatives) and 0.67 (experimenter generated alternatives) for the valuefluctuation model, 0.40 and 0.38 for the stochastic-dimension-selection 
model (Aschenbrenner et al., 1984). If one explains the missing positive correlation for described alternatives in the deterministic case with the finding that the processing orders vary randomly, the results are acceptable.

Again, described alternatives allow direct observation of the number of processed dimensions or features using the technique of sequentially uncovering information. According to our observations, the number of processed dimensions varies from choice to choice for about $75 \%$ of our subjects ( 21 out of 28 subjects in experiments 1,33 out of 42 subjects in experiment 2 in Schmalhofer et al., 1986).

According to the CDC model, the number of processed dimensions should increase with the similarity between choice alternatives. By definition, more similar alternatives have more features that are similar or even identical. Processing of the respective dimensions adds little or no attractiveness difference to the counter. Consequently, more dimensions must be processed before one of the critical values is reached and the process is terminated. The more similar two alternatives are, and the more identical features they have, the more dimensions should be inspected and processed on the average. This was found by Schmalhofer et al. (1986; Figure 2; experiment 1) to be true. Figures 3-3 (a) and 3-3 (b) present the respective new results of the two conditions (without and with consequences) of experiment 2 from Schmalhofer et al., which replicated the results of experiment 1.

The CDC models postulate termination and choice when the aggregated attractiveness values favoring one or the other alternative reach or exceed one of two corresponding criteria. A testable consequence of this assumption is that the most extreme counter value-taken absolutely-should always occur in the last processing step. Otherwise one criterion would have been reached earlier, and thus the choice process would have been terminated earlier. The technique of sequential uncovering allows the observation of the specific dimensions inspected and their sequence. Thus the counter value may be calculated from the attractiveness ratings for all processing steps and should be highest for the last dimension being processed. If, however, termination and choice were independent of reaching any criterion, the most extreme counter value could occur with equal probability at any of the observed processing steps. The prediction of the CDC model is tested here for the data of experiment 2 from Schmalhofer et al. (1986).

The clear bars in Figure 3-4 show how often (over all choice pairs and subjects) the most extreme counter value was observed in the last, next to last, and so on processing step. The striped bars show the frequencies that would be expected under the assumption of independent termination (they also differ in height due to varying numbers of inspected dimensions). The distribution of the observed counter values rejects the null-hypothesis that termination occurs independently of size of the counter value and favors the prediction of the CDC model, thus replicating former findings (Schmalhofer et al., 1986; experiment 1). 


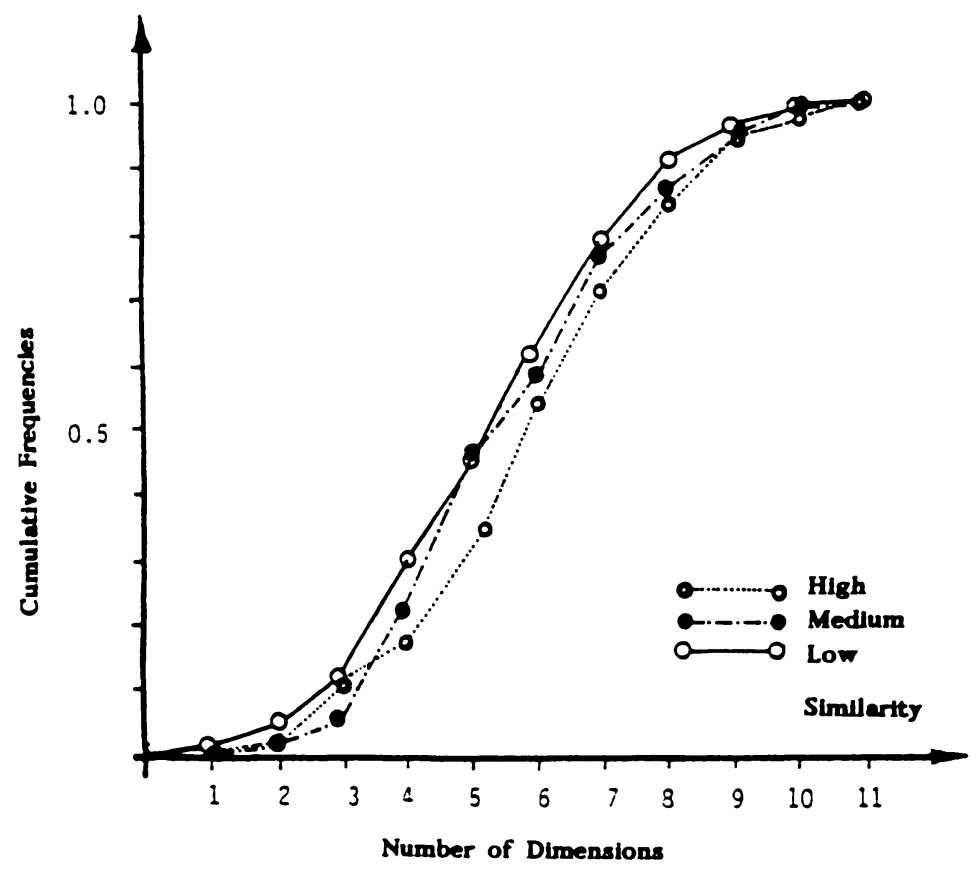

(a)

Figure 3-3. Cumulative frequency distributions of the number of inspected dimensions for three classes of choice pairs: (a) without and (b) with choice consequences.

\section{Comparison with Other Models}

In order to examine whether the CDC models are good candidates for modeling the process of choice for named and described alternatives, our research mainly focused on consequences specific to these models. Recently, a model comparison was performed. The predictions of different models able to predict binary choices must necessarily overlap to a considerable extent. Even if the predictions of two models would be made randomly, for example, by flipping two coins, both would correctly predict about $50 \%$ of the choices; thus a choice rule should predict substantially better. We would prefer that choice rule with the maximum number of correct predictions, in spite of the prediction overlap. Thus 15 more or less well-known choice rules that are able to handle binary choices were applied to the data provided by Schmalhofer et al. (1986; experiment 1; described alternatives, uncovering of features) by Albert, Aschenbrenner, Engemann, Radtke, Sachs, and Schmalhofer (submitted). Out of the 15 rules, 4 were variants of the CDC models. The most successful (78.57\% correct predictions) of these CDC variants was again the cardinal CDC model applied with known sequences of information processing and estimating the critical values independently of the 


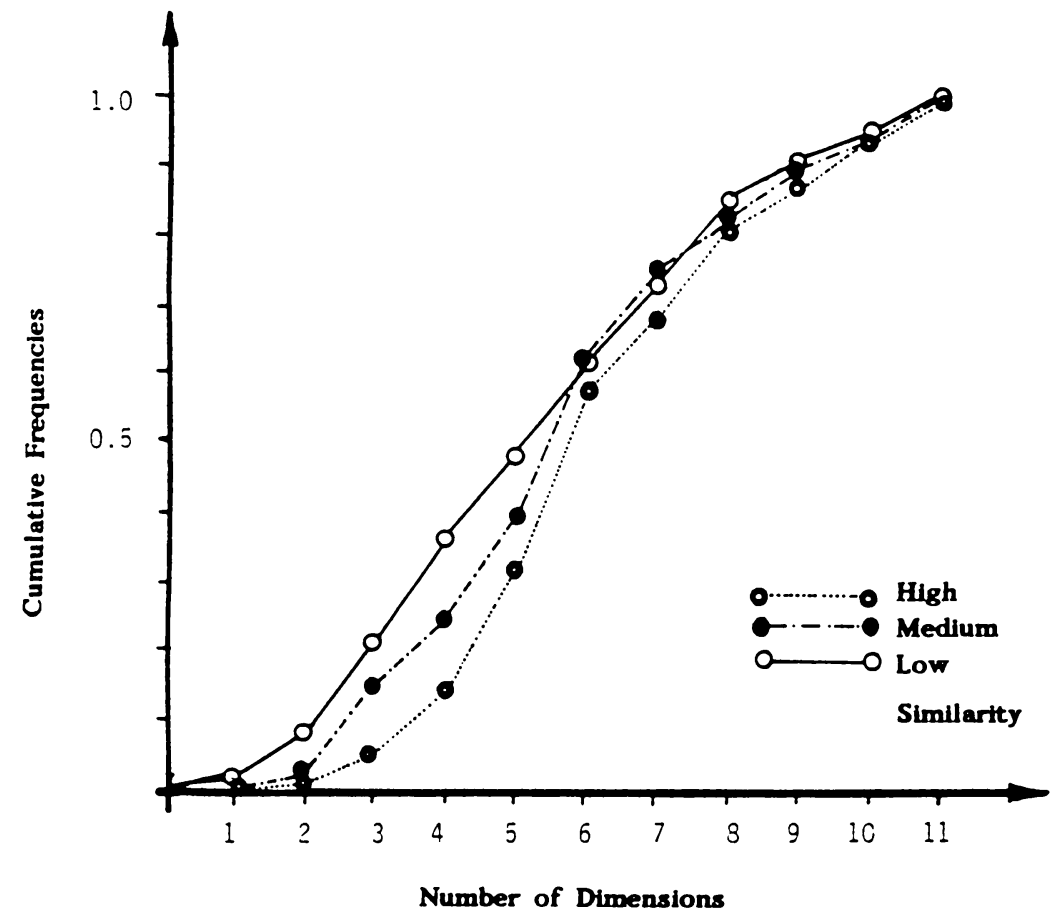

(b)

Figure 3-3. (Continued)

choices. The majority rule $(70.48 \%)$, weightpro rule $(77.38 \%)$ and the lexicoagraphic rule $(72.14 \%)$ also predicted over $70 \%$ correctly; the additive utility rule was even more successful than the best CDC variant with $79.05 \%$ correct predictions. However, only the CDC rule and, as submodel of it, the lexicographic rule allows for selective and adaptive information processing. While the other successful rules-including the additive utility rule-assume processing of the same dimensions for all pairs of alternatives. However, by observing the uncovered feature pairs we can clearly rule out this assumption, thus again questioning the percentage of correct predictions as the only criterion for model evaluation and favoring the CDC model in comparison to other models.

\section{Alternative Based Aggregation into Holistic Evaluations or Attitudes?}

According to the results discussed in the prior section, the additive utility model predicts choices very well, whereas other observations demonstrate violations of its processing assumptions. This clearly supports our initial 


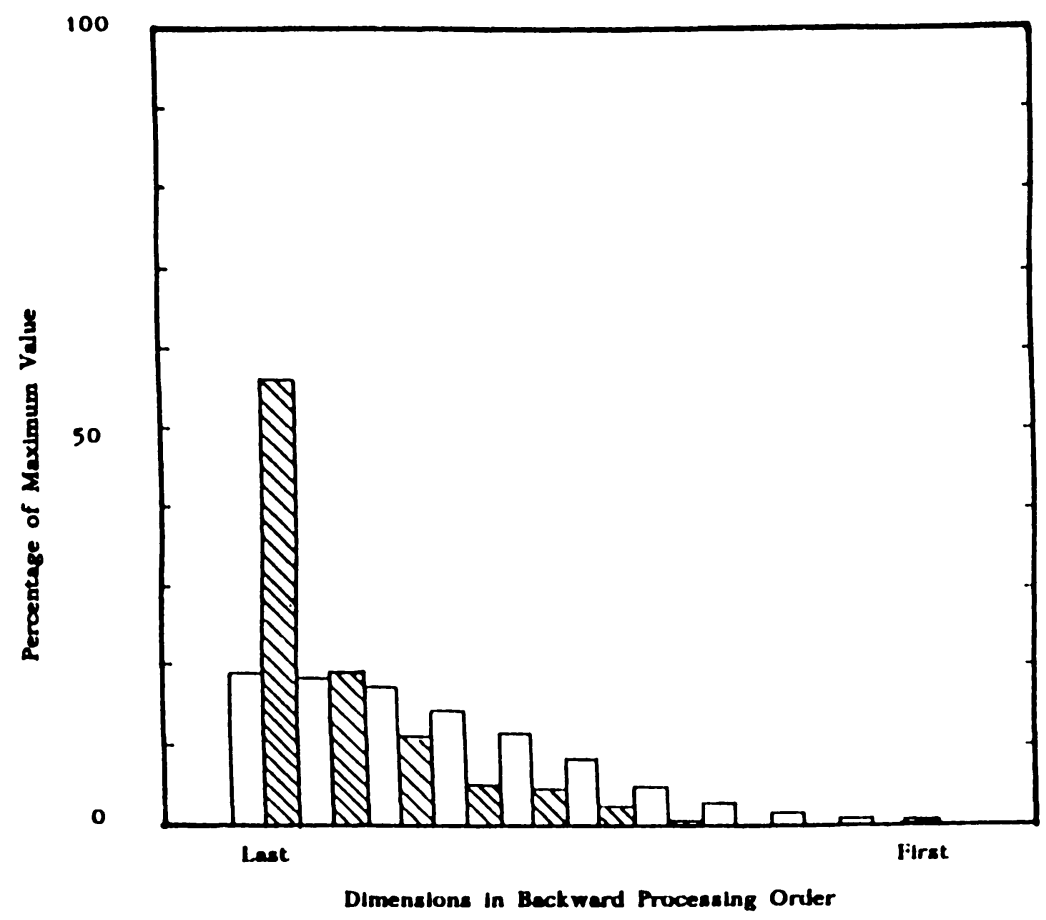

Figure 3-4. Frequency distributions of the maximum counter values for the 11 dimensions in backward processing order. Clear columns are the observed frequencies, striped columns are the frequencies that are predicted under the assumption of the null hypothesis.

scepticism about Ajzen and Fishbein's (1980) and Fishbein and Ajzen's (1975) results when interpreted as indicating characteristics of the choice process.

It might be argued, however, that many choices are not made between alternatives presented simultaneously, and that for alternatives presented sequentially an alternative based aggregation into holistic evaluations or attitudes with a subsequent comparison of these holistic evaluations may be more convenient (Tversky, 1969). This hypothesis has been investigated with described alternatives (text processing systems) by Schmalhofer and Gertzen (1986). In principle, the study adopted the evaluation part and the binary choice part for described alternatives with the following additional independent variation: the two alternatives of a choice pair were presented (1) simultaneously, (2) sequentially, or (3) sequentially with an interfering task in-between. By this variation, dimensional comparisons were made progressively more difficult, thus suggesting the use of alternative based processing strategies. Each description was presented together with the respective 
alternative's fictitious name. After the choice tasks, the subjects gave holistic evaluative judgments on a 9-point rating scale regarding the alternatives presented by names without the descriptions (memory judgments) and about the alternatives presented by their descriptions without the names (regular judgments).

If subjects base their decisions on holistic judgments, these should be incidentally learned by the subjects while choosing and then stored in memory. The memory judgments should therefore agree with the choices and with the holistic regular judgments of the descriptions.

The results (Table 3-7) indicate that for both of the two agreement scores a stronger tendency toward an alternative based processing strategy exists for sequential presentation; the tendency is even stronger for sequential presentation with an interpolated task between alternatives. However, the agreement scores were lower than expected. Therefore, a replication study has been performed by Gertzen and Schmalhofer (1986), the results of which are presented in Table 3-8.

The range of the agreement scores reappeared on a global level; but the expected increase of the agreement scores over conditions could not be replicated, thus questioning the previously discussed interpretation. In order to get more insight into the processing of information, a think-aloud study was performed for the sequential presentation condition using Schmalhofer and Schäfer's (1986) CDC model based category system including an overall judgment category. The results from 120 binary choices (Table 3-9) show that there was indeed a substantial amount of overall judgments (104 utterances), namely for approximately each second alternative (of the 240 alternatives presented). There was also a considerable amount of dimensional processing (96 utterances) which was only possible when the second alternative was available and which obviously reduced alternative based processing of the second alternative.

The results do indicate, however, that subjects may use dimensional as well as alternative based processing in choosing between sequentially presented alternatives.

For simultaneous presentation of alternatives, think-aloud data of Schmalhofer and Schäfer (1986) indicate some amount of holistic evaluation

Table 3-7. Relative Frequency of Correct Choice Predictions by Judgments from Memory, and Correlations Between Judgments from Memory and Regular Judgments

\begin{tabular}{lccc}
\hline & \multicolumn{3}{c}{ Presentation of Alternatives } \\
\cline { 2 - 4 } & Simultaneous & Sequential & With Interpolation Task \\
\hline Predictions & .54 & .65 & .73 \\
Correlations & .22 & .27 & .35 \\
\hline
\end{tabular}

From Schmalhofer \& Gertzen (1986), with permission. 
Table 3-8. Relative Frequency of Correct Choice Predictions by Judgments from Memory, and Correlations Between Judgments from Memory and Regular Judgments

\begin{tabular}{lccc}
\hline & \multicolumn{3}{c}{ Presentation of Alternatives } \\
\cline { 2 - 4 } & Simultaneous & Sequential & With Interpolation Task \\
\hline Predictions & .71 & .63 & .69 \\
Correlations & .25 & .23 & .36 \\
\hline
\end{tabular}

From Gertzen \& Schmalhofer (1986), with permission.

for named alternatives ( $15 \%$ of utterances), while almost none for described alternatives ( $2 \%$ of utterances).

Taken together, alternative based aggregation rules in the sense of Ajzen and Fishbein may be used under some conditions for more than some choices, but as a general rule this was not found for binary choice; contradicting Ajzen and Fishbein's reasoning.

\section{General Discussion}

As argued at the start of this chapter, Fishbein and Ajzen consider their theory of reasoned action as a process model for the prediction of various kinds of behavioral choices. Models of this kind are indeed well suited for the prediction of choices, as is also shown by our data. The processes that precede behavioral choice are, nevertheless, inadequately described by models of the Fishbein and Ajzen type. At least for binary choice the CDC models appear more appropriate. Each individual finding that supports the process assumptions of the CDC models may be subject to alternative

Table 3-9. Percentages of Four Different Verbalizations and a Rest Category for the First and Second Presented Choice Alternative ${ }^{a}$

\begin{tabular}{lccc}
\hline & \multicolumn{2}{c}{ Alternative } & \\
\cline { 2 - 3 } & First & Second & Total \\
\hline Single features & $43 \%$ & $23 \%$ & $70 \%$ \\
Dimensional comparisons & - & $11 \%$ & $11 \%$ \\
Overall judgments & $7 \%$ & $5 \%$ & $12 \%$ \\
Mentioning of dimensions & $1 \%$ & $1 \%$ & $2 \%$ \\
Rest category & $1 \%$ & $4 \%$ & $5 \%$ \\
\hline
\end{tabular}

From Gertzen \& Schmalhofer (1986), with permission.

${ }^{\text {a }}$ For 120 analyzed choice trials, the total number of verbalizations was 870 . 
interpretation. But taken jointly the empirical evidence appears to support the CDC models considerably.

We do not claim the same degree of universality for the CDC models as was done with the theory of reasoned action and its successors. The following are our reasons for this belief.

1. Thus far the CDC models have been tested solely in the laboratory. Field tests are still under way. Currently, the theory is applied to delivery choices of pregnant women. But even such (hopefully) successful applications will probably not result in the replacement of former prediction models by the CDC models. It is doubtless that the CDC models are well suited for prediction whenever they describe the underlying processes. However, the effort that is required for the assessment and estimation of the model parameters will usually not be justified if prediction is the sole objective.

2. Up to now CDC models have been designed and tested solely for binary-forced choice. We do not expect the same processes for all other kinds of behavioral choice. Rather, it is to be expected that further variations in the choice situation will influence the process components in similar ways, as was shown for the variation of presentation modes. For example, the latter variation from named to described presentation influenced the subprocess of information selection, whereas other subprocesses remained the same. Generally, we suggest that cognitive choice processes adapt their components flexibly to task as well as to situational characteristics.

This selectivity and adaptivity of cognitive choice processes has two consequences for the attitude-behavior relationship: (1) The principles that underlie this adaptation performance should be studied more thoroughly. (2) The more basic processes of evaluation which, as we see it, are basic to all cognitive choice processes should be studied more thoroughly.

Acknowledgments. The research presented here was supported by a grant from the Deutsche Forschungsgemeinschaft (Al 205/1); Schwerpunktprogramm "Einstellung und Verhalten."

We gratefully acknowledge the assistance of Maria Bannert, Ulf Böckenholt, Joachim Canisius, Heiner Gertzen, Doris Glavanov, Joachim Hecker, Otto Kühn, Roland Laier, Isabelle Müller, Waltrun Noe, Walter Saffrich, Iris Schäfer, Thomas Schneyer, and Manfred Stickel. The benefits of critical discussions with Hans Kordy are also appreciated.

Requests for reprints should be sent to Dietrich Albert, Psychologisches Institut, Universität Heidelberg, Hauptstrasse 47-51, D-6900 Heidelberg, Federal Republic of Germany.

\section{References}

Ajzen, I. (1985). From intentions to actions: A theory of planned behavior. In J. Kuhl, \& J. Beckmann, (Eds.), Action control: From cognition to behavior (pp. 11-39). Berlin: Springer.

Ajzen, I., \& Fishbein, M. (1977). Attitude-behavior relations: A theoretical analysis and review of empirical research. Psychological Bulletin, 84, 888-918. 
Ajzen, I., \& Fishbein, M. (1980). Understanding attitudes and predicting social behavior. Englewood Cliffs, NJ: Prentice-Hall.

Albert, D. (1968). Freies Reproduzieren als stochastische Entleerung eines Speichers. Zeitschrift für experimentelle und angewandte Psychologie, 15, 564-581.

Albert, D., Aschenbrenner, K.M., Engemann, A., Radtke, M., Sachs, S., \& Schmalhofer, F. (submitted). Individuelle Binärwahlen: Ein Vergleich von simulierten Wahlheuristiken.

Albert, D., Schmalhofer, F., \& Aschenbrenner, K.M. (1983, August). A Markovian model of individual choice behavior. Paper presented at the 16th Annual Mathematical Psychology Meeting, Boulder, CO.

Albert, D., \& Schulz, U. (1976). Das Reproduzieren und seine Beendigung: Ein Ausschöpfmodell. Psychologische Beiträge, 18, 1-11.

Albert, D., \& Schulz, U. (1981). Kritischer Test eines Modells zum Reproduzieren von Einheiten mehrerer Klassen durch Einbeziehung der Reproduktionszeiten oder: Über die Konkurrenz von Reproduktionstendenzen. In L. Tent (Hrg.), ErkennenWollen-Handeln (pp. 67-86). Göttingen/Toronto/Zürich: Hogrefe.

Aschenbrenner, K.M. (1979). Kompexes Wahlverhalten als Problem der Informationsverarbeitung. In H. Ueckert \& D. Rhenius (Hrg.), Komplexe menschliche Informationsverarbeitung (pp. 411-424). Bern/Stuttgart/Wien: Huber.

Aschenbrenner, K.M. (1984). Moment- versus dimension-oriented theories of risky choice: A (fairly) general test involving single-peaked preferences. Journal of Experimental Psychology: Learning, Memory, and Cognition, 10, 315-335.

Aschenbrenner, K.M. (1985). Das Eingipfligkeitsphänomen: Seine Erklärung und Bedeutung. In D. Albert (Hrg.), Bericht über den 34. Krongress der Deutschen Gesellschaft für Psychologie in Wien 1984, Bd. 1, Grundlagenforschung (pp. 49-54). Göttingen: Hogrefe.

Aschenbrenner, K.M., Albert, D., \& Schmalhofer, F. (1984). Stochastic choice heuristics. Acta Psychologica, 56, 153-166.

Aschenbrenner, K.M., Bockenholt, U., Albert, D., \& Schmalhofer, F. (1986). The selection of dimensions when choosing between multi-attribute alternatives. In R.W. Scholz (Ed.), Current issues in West-German decision research (pp. 63-78). Frankfurt: Lang.

Audley, R.J. (1963). A stochastic model for individual choice behavior: In R.D. Luce, R.R. Bush, \& E. Galanter (Eds.), Readings in mathematical psychology (vol. 1, pp. 263-277). New York: Wiley.

Audley, R.J., \& Pike, A.R. (1965). Some alternative stochastic models of choice. British Journal of Mathematical and Statistical Psychology, 18, 207-225.

Birnbaum, M.H. (1973). The devil rides again: Correlation as an index of fit. Psychological Bulletin, 79, 239-242.

Bower, G.H. (1959). Choice-point behavior. In R.R. Bush \& W.K. Estes (Eds.), Studies in mathematical learning theory (pp. 109-124). Stanford, CA: Stanford University Press.

Busemeyer, J.R. (1982). Choice behavior in a sequential decision making task. Organizational Behavior and Human Performance, 29, 175-207.

Busemeyer, J.R. (1985). Decision making under uncertainty: A comparison of simple scalability, fixed-sample and sequential-sampling models. Journal of Experimental Psychology: Learning, Memory, and Cognition, 11, 538-564.

Busemeyer, J.R., \& Jones, L.E. (1983). Analysis of multiplicative combination rules. When the causal variables are measured with error. Psychological Bulletin, 93, 549562.

Cronen, V.E., \& Conville, R.E. (1975). Fishbein's conception of belief strength: A theoretical, methodological and experimental critique. Speech Monographs, 42, 143-150.

Dawes, R.M. (1979). The robust beauty of improper linear models in decision making. American Psychologist, 34, 571-582. 
Dawes, R.M., \& Corrigan, B. (1974). Linear models in decision making. Psychological Bulletin, 81, 95-106.

Delia, J., Crockett, W., Press, A., \& O'Keefe, D. (1975). The dependency of interpersonal evaluations on context-relevant beliefs about others. Speech Monographs, 42, 10-19.

Einhorn, H.J., Kleinmuntz, D.N., \& Kleinmuntz, B. (1979). Linear regression and process-tracing models of judgment. Psychological Review, 5, 465-485.

Estes, W.K. (1959). A random walk model for choice behavior. In K.J. Arrow, S. Karlin, \& P. Suppes (Eds.), Mathematical methods in the social sciences (pp. 265-276). Stanford, CA: Stanford University Press.

Feller, W. (1957). An introduction to probability theory and its applications. (vol. 1, 2nd ed.). New York: Wiley.

Fishbein, M. (1963). An investigation of the relationship between beliefs about an object and the attitude toward that object. Human Relations, 16, 233-240.

Fishbein, M. (1967). A behavior theory approach to the relations between beliefs about an object and the attitude toward the object. In M. Fishbein (Ed.), Readings in attitude theory and measurement (pp. 389-400). New York: Wiley.

Fishbein, M. (1973). The prediction of behavior from attitudinal variables. In C.D. Mortensen \& K.K. Sereno (Eds.), Advances in communication research (pp. 3-31). New York: Harper \& Row.

Fishbein, M., \& Ajzen, I. (1974). Attitudes toward objects as predictors of single and multiple behavioral criteria. Psychological Review, 81, 59-74.

Fishbein, M. \& Ajzen, I. (1975). Belief, attitude, intention and behavior: An introduction to theory and research. Reading, MA: Addison-Wesley.

Gertzen, H., \& Schmalhofer, F. (1986). Cognitive choice processes for sequentially or simultaneously presented alternatives. In R.W. Scholz (Ed.), Current issues in WestGerman decision research (pp. 79-94). Frankfurt: Lang.

Krantz, D.H., Luce, R.D., Suppes, P., \& Tversky, A. (1971). Foundations of measurement. Vol. 1: Additive and polynomial representations. New York: Academic Press.

Kühn, O., Schmalhofer, F., Albert, D., \& Aschenbrenner, K.M. (1986, March). Der Einfluss der Betrachtungsperspektive auf Textkodierung, Gedächtnisabruf und Entscheidungsfindung. Vortrag auf der 28. Tagung experimentell arbeitender Psychologen (TEAP), Saarbrücken.

Laier, R., Albert, D., Schmalhofer, F., \& Aschenbrenner, K.M. (1986, March). Haben Absoluturteile (Ratings) über Lange und Attraktivitat Intervallskalenniveau? Vortrag auf der 28. Tagung experimentell arbeitender Psychologen (TEAP), Saarbrücken.

Lichtenstein, S., \& Slovic, P. (1971). Reversals of preference between bids and choices in gambling decisions. Journal of Experimental Psychology, 89, 46-55.

Link, S.W. (1978). The relative judgment theory of the psychometric function. In J. Reguin (Ed.), Attention and performance vii (pp. 619-630). New York: Wiley.

Link, S.W., \& Heath, R.A. (1975). A sequential theory of psychological discrimination. Psychometrika, 40, 77-105.

Luce, R.D. (1959). Individual choice behavior: A theoretical analysis. New York: Wiley.

Luce, R.D. (1977). The choice axiom after twenty years. Journal of Mathematical Psychology, 15, 215-233.

Müller, G.E., \& Pilzecker, A. (1900). Experimentelle Beiträge zur Lehre vom Gedächtnis. Zeitschrift für Psychologie, Ergänzungsband 1, Leipzig: Barth.

Orth, B. (1979). Rating-Verfahren und Grössenschätz-Methode: Skalenniveau und funktionale Zusammenhänge zwischen Skalen. Diss. Math.-Naturwiss. Fak., Univ. Kiel.

Orth, B. (1982). Zur Bestimmung der Skalenqualität bei ,direkten' Skalierungsverfahren. Zeitschrift für experimentelle und angewandte Psychologie, 24, 160-178.

Payne, J.W. (1982). Contingent decision behavior. Psychological Bulletin, 9, 382-402.

Petrusic, W.M., \& Jamieson, D.G. (1978). Relation between probability of preferential 
choice and time to choose changes with practice. Journal of Experimental Psychology: Human Perception and Performance, 4, 471-482.

Raijmakers, J.G.W., \& Shiffrin, R.M. (1981). Search of associative memory. Psychological Review, 88, 93-134.

Ratcliff, R. (1978). A theory of memory retrieval. Psychological Review, 85, 59-108.

Russo, J., \& Dosher, B.A. (1983). Strategies for multiattribute binary choice. Journal of Experimental Psychology: Learning, Memory, and Cognition, 9, 676-696.

Schmalhofer, F. (1987). Expert systems as cognitive tools for human decision making. In J.L. Mumpower, L.E. Phillips, O. Renn, \& V.R.R. Uppuluri (Eds.), Expert judgment and expert systems (pp. 269-288). Berlin, Heidelberg: Springer.

Schmalhofer, F., Albert, D., Aschenbrenner, K.M., \& Gertzen, H. (1986). Process traces of binary choices: Evidence for selective and adaptive decision heuristics. The Quarterly Journal of Experimental Psychology, 38A, 59-76.

Schmalhofer, F., Aschenbrenner, K.M., Albert, D., \& Gertzen, H. (submitted). Criterion-dependent choices between binary alternatives presented by name or multidimensional description.

Schmalhofer, F., \& Gertzen, H. (1986). Judgment as a component decision process for choosing between sequentially available alternatives. In B. Brehmer, H. Jungermann, P. Lourens, \& G. Sevón (Eds.), New directions in research on decision making (pp. 139-150). North-Holland: Elsevier.

Schmalhofer, F. \& Saffrich, W. (1984, August). Effort-quality trade-off characteristics of selective information processing in binary choices. Unpublished manuscript of a paper presented at the European Mathematical Psychology Meeting, Utrecht/Holland.

Schmalhofer, F., \& Schäfer, I. (1986). Lautes Denken bei der Wahl zwischen benannt und beschrieben dargebotenen Alternativen. Sprache und Kognition, 2, 7381.

Schoemaker, P.J.H. (1979). The role of statistical knowledge in gambling decisions: Moment vs. risk dimension approaches. Organizational Behavior and Human Performance, 24 (1), 1-17.

Schoemaker, P.J.H., \& Waid, C.C. (1982). An experimental comparison of different approaches to determining weights in additive utility models. Management Science, 28, 101-120.

Shiffrin, P.M. (1970). Memory search. In D.A. Norman (Ed.), Models of memory (pp. 375-447). New York: Academic Press.

Six, B. (1980). Das Konzept der Einstellung und seine Relevanz für die Vorhersage des Verhaltens. In F. Petermann (Hrg.), Einstellungsmessung-Einstellungsforschung (pp. 55-84). Göttingen: Hogrefe.

Stroebe, W. (1980). Grundlagen der Sozialpsychologie I. Stuttgart: Klett-Cotta.

Strube, G. (1984). Assoziation: der Prozess des Erinnerns und die Struktur des Gedächtnisses. Lehr- und Forschungstexte Psychologie, Bd. 6. Berlin/New York/Heidelberg: Springer.

Svenson, O. (1979). Process descriptions of decision making. Organizational Behavior and Human Performance, 23, 86-112.

Thorngate, W. (1980). Efficient decision heuristics. Behavioral Science, 25, 219-225.

Thurstone, L.L. (1927). A law of comparative judgment. Psychological Review, 34, 273286.

Tversky, A. (1969). Intransitivity of preferences. Psychological Review, 76, 31-48.

Upmeyer, A. (1982). Attitudes and social behavior. In J.P. Codol, \& J.-P. Leyens, (Eds.), Cognitive analysis of social behavior (pp. 51-86). The Hague: Nijhoff.

van Putten, W.L.J. (1982). Maximum likelihood estimates for Luce's choice model. Journal of Mathematical Psychology, 25, 163-174.

Wald, A. (1950). Sequential analysis. New York: Wiley.

Wallsten, T.S. (1980). Processes and models to describe choice and inference behavior. In T.S. Wallsten (Ed.), Cognitive processes in choice and decision behavior (pp. 215-237). Hillsdale, NJ: Erlbaum. 
Wallsten, T.S., \& Barton, C. (1982). Processing probabilistic multidimensional information for decisions. Journal of Experimental Psychology: Learning, Memory, and Cognition, 8, 361-384.

Wicker, A.W. (1969). Attitudes versus actions: The relationship of verbal and overt behavioral responses to attitude objects. Journal of Social Issues, 25, 41-78. 